\title{
ON FIXED POINT PLANAR ALGEBRAS
}

\author{
By ARnaud Brothier!
}

\begin{abstract}
To a weighted graph can be associated a bipartite graph planar algebra $\mathcal{P}$. We construct and study the symmetric enveloping inclusion of $\mathcal{P}$. We show that this construction is equivariant with respect to the automorphism group of $\mathcal{P}$. We consider subgroups $G$ of the automorphism of $\mathcal{P}$ such that the $G$-fixed point space $\mathcal{P}^{G}$ is a subfactor planar algebra. As an application we show that if $G$ is amenable, then $\mathcal{P}^{G}$ is amenable as a subfactor planar algebra. We define the notions of a cocycle action of a Hecke pair on a tracial von Neumann algebra and the corresponding crossed product. We show that a large class of symmetric enveloping inclusions of subfactor planar algebras can be described by such a crossed product.
\end{abstract}

\section{INTRODUCTION AND MAIN RESULTS}

The theory of subfactors has been initiated by Jones [13]. Given a subfactor (an extremal unital inclusion of factors of type $\mathrm{II}_{1}$ with finite index), Jones associated a combinatorial invariant called the standard invariant. It has been axiomatized in the finite depth case as a paragroup by Ocneanu [22. Then, it has been axiomatized in the general case as a $\lambda$-lattice by Popa [29] and as a subfactor planar algebra by Jones [12]. The reconstruction theorem of Popa shows that any $\lambda$-lattice is the standard invariant of a subfactor, see [26, 29, 31].

Popa studied analytic properties of subfactors and among other defined the notion of amenability in this context [27]. He gave many characterizations of amenability and defined it for $\lambda$-lattices and thus for subfactor planar algebras. He constructed the socalled symmetric enveloping inclusion $T \subset S$ associated to a subfactor $N \subset M$ [28] which extends constructions due to Ocneanu, and Longo and Rehren [22, 19]. The symmetric enveloping inclusion is a subfactor of type $\mathrm{II}_{1}$ and has finite index if and only if $N \subset M$ has finite depth. Let $\mathcal{P}$ be the subfactor planar algebra of a subfactor $N \subset M$. Popa proved in [30] that $\mathcal{P}$ is amenable if and only if the symmetric enveloping inclusion of $N \subset M$ is co-amenable, see Section 1.3 .

Given a subfactor planar algebra $\mathcal{Q}$, one can construct a tower of $\mathrm{II}_{1}$ factors $M_{0} \subset$ $M_{1} \subset M_{2} \subset \cdots$ and a sequence of symmetric enveloping inclusions $T_{k} \subset S_{k}, k \geqslant 0$ [11, 8]. It has been proven that the standard invariant of $M_{0} \subset M_{1}$ is the subfactor planar algebra $\mathcal{Q}$ and the symmetric enveloping inclusion of $M_{k-1} \subset M_{k}$ is isomorphic to the inclusion $T_{k} \subset S_{k}$ for any $k \geqslant 1$. We call $T_{0} \subset S_{0}$ the symmetric enveloping inclusion associated to $\mathcal{Q}$.

Given a bipartite graph $\Gamma$ and a weight $\mu$ on its edges satisfying certain assumptions we can construct a planar algebra $\mathcal{P}$ called a bipartite graph planar algebra [14, 7]. See also [21]. The automorphism group $\operatorname{Aut}(\mathcal{P})$ of $\mathcal{P}$ is isomorphic to a semi-direct product $U\left(\mathcal{P}_{1}^{+}\right) \rtimes \operatorname{Aut}(\Gamma, \mu)$ where $\operatorname{Aut}(\Gamma, \mu)$ is the group of automorphisms of $\Gamma$ that preserves the weight $\mu$ and $U\left(\mathcal{P}_{1}^{+}\right)$is the unitary group of the 1-box space $\mathcal{P}_{1}^{+}$. The group $\operatorname{Aut}(\mathcal{P})$

\footnotetext{
${ }^{1}$ Department of Mathematics, University of Rome Tor Vergata, Via della Ricerca Scientifica, 1 - 00133 Roma, Italy, arnaud.brothier@gmail.com, https://sites.google.com/site/arnaudbrothier/
} 
acts on the vertices $V=V^{+} \cup V^{-}$of $\Gamma$ where $U\left(\mathcal{P}_{1}^{+}\right)$acts trivially. If $G<\operatorname{Aut}(\mathcal{P})$ is any subgroup, then the fixed point space $\mathcal{P}^{G}$ is a planar algebra. If $G$ acts transitively on the even and odd vertices of $\Gamma$, then $\mathcal{P}^{G}$ satisfies automatically all the axioms of a subfactor planar algebra except sphericality. In particular we obtain that $\mathcal{P}^{G}$ is non-degenerate which is usually the hardest axiom to check for a subfactor planar algebra, see Section 1 for definitions. Note that starting from a bipartite graph $\Gamma$ and a vertex-transitive group action $G \curvearrowright \Gamma$, there exists a unique weight $\mu$ such that we can associate to $(\Gamma, \mu)$ a bipartite graph planar algebra $\mathcal{P}$ and such that the fixed point space $\mathcal{P}^{G}$ is a subfactor planar algebra, see [2, Proposition 2.5].

In this article, we consider bipartite graph planar algebras $\mathcal{P}$ and their planar subalgebras $\mathcal{Q} \subset \mathcal{P}$. We investigate the structure of $\mathcal{Q}$, its symmetric enveloping inclusion, and the notion of amenability when $\mathcal{Q}$ is a subfactor planar algebra embedded in $\mathcal{P}$. We start by proving that $\mathcal{Q}$ is non-amenable if $\|\Gamma\|<\delta$, where $\delta$ is the modulus of $\mathcal{Q}$. The proof is elementary and is independent from the rest of the paper. We extend the construction of [11, 8] and associate to a bipartite graph planar algebra $\mathcal{P}$ a tower of von Neumann algebras $M_{0} \subset M_{1} \subset M_{2} \subset \cdots$ and a sequence of inclusions $T_{k} \subset S_{k}, k \geqslant 0$. Note that the construction of the tower was already explained in [11, Section 4]. We prove that $M_{i}$ and $T_{k}$ are von Neumann algebras of type $\mathrm{II}_{1}$ with atomic centers and that $S_{k}$ is a factor of type II for any $i, k \geqslant 0$. We show that the automorphism $\operatorname{group} \operatorname{Aut}(\mathcal{P})$ of $\mathcal{P}$ acts in a minimal way on those von Neumann algebras and show that the constructions of $M_{i}$ and $S_{k}$ behaves as expected with respect to inclusions of planar algebras and group actions. In particular, if $G<\operatorname{Aut}(\mathcal{P})$ is a subgroup such that $\mathcal{P}^{G}$ is a subfactor planar algebra, then the inclusion of fixed point spaces $M_{0}^{G} \subset M_{1}^{G}$ is a subfactor with standard invariant isomorphic to $\mathcal{P}^{G}$ and the symmetric enveloping inclusion of this subfactor (resp. of $\mathcal{P}^{G}$ ) is isomorphic to $M_{1}^{G} \vee\left(M_{1}^{\mathrm{op}}\right)^{G} \subset S_{1}^{G}$ (resp. $\left.M_{0}^{G} \vee\left(M_{0}^{\mathrm{op}}\right)^{G} \subset S_{0}^{G}\right)$. As an application we prove the following theorem.

Theorem A. Consider a bipartite graph planar algebra $\mathcal{P}$ over a weighted graph $(\Gamma, \mu)$ and a subgroup $G<A u t(\mathcal{P})$ such that $\mathcal{P}^{G}$ is a subfactor planar algebra. If the group $G$ is amenable (as closed subgroup or a countable discrete group), then the subfactor planar algebra $\mathcal{P}^{G}$ is amenable.

Note that a more general framework has been studied independently by Arano and Vaes from which this theorem follows [2]. Recall that a Hecke pair $(G, H)$ is an inclusion of groups $H<G$ which is almost normal, i.e. for any $g \in G$ the group $H \cap g H g^{-1}$ has finite index inside $H$ and $\mathrm{gHg}^{-1}$. We define the notion of a cocycle action of a Hecke pair on a tracial von Neumann algebra and the corresponding twisted crossed product von Neumann algebra. Note that it has been considered already in the framework of ordinary action on $C^{*}$-algebras by Palma, see [23, 24]. We show that if $\mu$ is constant on the set of even edges and $G<\operatorname{Aut}(\mathcal{P})$ is a subgroup such that $\mathcal{P}^{G}$ is a subfactor planar algebra, then the symmetric enveloping inclusion of $\mathcal{P}^{G}$ can be described in the following way.

Theorem B. Consider a bipartite graph planar algebra $\mathcal{P}$ over a weighted graph $(\Gamma, \mu)$ such that $\mu$ is constant on the set of even edges and a subgroup $G<A u t(\mathcal{P})$. Assume that $\mathcal{P}^{G}$ is a subfactor planar algebra. Denote by $G_{o}$ the subgroup of $G$ that fixes an even vertex o of $\Gamma$. There exists a $I I_{1}$ factor $A$ and a cocycle action of the Hecke pair $\left(G, G_{o}\right)$ on $A \bar{\otimes} A^{o p}$ such that $G_{o}$ acts on $A$ and such that the symmetric enveloping inclusion of $\mathcal{P}^{G}$ is isomorphic to the inclusion $A^{G_{o}} \bar{\otimes}\left(A^{o p}\right)^{G_{o}} \subset v N\left[A \bar{\otimes} A^{o p} ; G, G_{o}\right]$, where $v N\left[A \bar{\otimes} A^{o p} ; G, G_{o}\right]$ is a twisted crossed product of $A \bar{\otimes} A^{o p}$ by the Hecke pair $\left(G, G_{o}\right)$. 
See Theorem 4.5 for a slightly more precise statement. Many symmetric enveloping inclusion of subfactors can be described in that way including diagonal and Bisch-Haagerup subfactors, see Section 4.3. This theorem can be interpreted as an extension of a theorem of Popa which shows that the symmetric enveloping algebra $S$ of a diagonal subfactor is the twisted crossed product of a von Neumann algebra by a group [27, Section 3].

\section{Preliminaries And A CRITERION OF NON-AMENABility}

A planar algebra is a collection of complex $*$-algebras $\mathcal{P}=\left(\mathcal{P}_{n}^{ \pm}: n \geqslant 0\right)$ on which the operad of shaded planar tangles acts. See [12, 15] for more details. We follow similar conventions to [8] for drawing a shaded planar tangle. We decorate strings with natural numbers to indicate that they represent a given number of parallel strings. The distinguished interval of a box is decorated by a dollar sign if it is not at the top left corner. We do not draw the outside box and will omit unnecessary decorations. The left and right traces of a planar algebra are the maps $\tau_{l}: \mathcal{P}_{n}^{ \pm} \longrightarrow \mathcal{P}_{0}^{\mp}$ and $\tau_{r}: \mathcal{P}_{n}^{ \pm} \longrightarrow \mathcal{P}_{0}^{ \pm}$defined for any $n \geqslant 0$ such that

$$
\tau_{l}(x)=\circlearrowleft \text { and } \tau_{r}(x)=x \text { for any } x \in \mathcal{P}_{n}^{ \pm} .
$$

Suppose that $\mathcal{P}_{0}^{ \pm}=\mathbb{C}$. The planar algebra is called spherical if the two traces agree on each element of $\mathcal{P}$. We say that $\mathcal{P}$ is non-degenerate if the sesquilinear forms $(x, y) \mapsto \tau_{l}\left(x y^{*}\right)$ and $(x, y) \mapsto \tau_{r}\left(x y^{*}\right)$ are positive definite. A subfactor planar algebra is a planar algebra such that each space $\mathcal{P}_{n}^{ \pm}$is finite dimensional, $\mathcal{P}_{0}^{ \pm}=\mathbb{C}, \mathcal{P}$ is spherical, and is nondegenerate. The modulus of a subfactor planar algebra is the value of a closed loop. The index of a subfactor planar algebra is the square of its modulus. Note that all the subfactors considered in this article are extremal. This condition corresponds to the sphericality of their associated subfactor planar algebra. '

1.1. Bipartite graph planar algebras. We refer to [14] and [7] for more details about bipartite graph planar algebras of finite and infinite graphs respectively. Let $\Gamma$ be a countable locally finite undirected connected bipartite graph that can have multiple edges between two vertices. Denote by $V=V^{+} \sqcup V^{-}$its set of vertices, where $V^{+}$and $V^{-}$are the set of even and odd vertices respectively. We consider the associated symmetric oriented graph obtained by doubling each edge of $\Gamma$ into a pair of oppositely oriented edges. If $a$ is a path, then we denote by $\bar{a}, s(a), t(a)$ its associated opposite edge, its source, and its target respectively.

We put $C_{0}^{ \pm}=V^{ \pm}, C_{n}^{ \pm}, n \geqslant 1$ the set of paths in $\Gamma$ of length $n$ that start in $V^{ \pm}, C_{*}^{ \pm}=$ $\bigcup_{n \geqslant 0} C_{n}^{ \pm}$. Consider the Hilbert space $\ell^{2}\left(C_{n}^{ \pm}\right)$with orthonormal basis indexed by $C_{n}^{ \pm}$. Let $B\left(\ell^{2}\left(C_{n}^{ \pm}\right)\right)$be the space of bounded linear operators on $\ell^{2}\left(C_{n}^{ \pm}\right)$with the standard system of matrix units $\left\{e_{a, b}: a, b \in C_{n}^{ \pm}\right\}$. Define the von Neumann subalgebra $\mathcal{P}_{n}^{ \pm} \subset B\left(\ell^{2}\left(C_{n}^{ \pm}\right)\right)$ generated by the operators $e_{a, b}$ where $a, b$ are in $C_{n}^{ \pm}$and have the same source and target. We denote by $S T_{n}^{ \pm}, n \geqslant 0$ the set of couples $(a, b)$ where $a, b \in C_{n}^{ \pm}, s(a)=s(b)$, and $t(a)=t(b)$. We put $S T_{*}^{ \pm}=\cup_{n \geqslant 0} S T_{n}^{ \pm}$. Observe that the von Neumann algebra $\mathcal{P}_{0}^{ \pm}$is isomorphic to the abelian atomic von Neumann algebra of bounded functions $\ell^{\infty}\left(V^{ \pm}\right)$. We identify $\mathcal{P}_{0}^{ \pm}$with $\ell^{\infty}\left(V^{ \pm}\right)$. We denote by $\left\{e_{v}: v \in V^{ \pm}\right\}$the set of minimal projections of $\mathcal{P}_{0}^{ \pm}$.

Assume there exists a strictly positive map $\mu: C_{1} \longrightarrow \mathbb{R}_{+}^{*}$ called a weight satisfying that for any paths $a=a_{1} \cdots a_{n}, b=b_{1} \cdots b_{l}$ of length $n, l \geqslant 1$ such that $s\left(a_{1}\right)=s\left(b_{1}\right), t\left(a_{n}\right)=$ 
$t\left(b_{l}\right)$ we have that

$$
\begin{gathered}
\qquad \mu\left(a_{1}\right) \cdots \mu\left(a_{n}\right)=\mu\left(b_{1}\right) \cdots \mu\left(b_{l}\right) \text { and } \\
\text { there exists } \delta>0 \text { such that } \sum_{c \in C_{1}: s(c)=v} \mu(c)=\delta \text { for any } v \in V .
\end{gathered}
$$

Such a pair $(\Gamma, \mu)$ is called a weighted graph with modulus $\delta$ or simply a weighted graph. Note that a weaker notion has been introduced and studied in [9] where the first assumption is replaced by $\mu(a) \mu(\bar{a})=1$ for any edge $a$ where $\bar{a}$ denotes the opposite edge of $a$.

Recall that the degree $\operatorname{deg}(v)$ of a vertex $v$ is the number of edges with source $v$ and

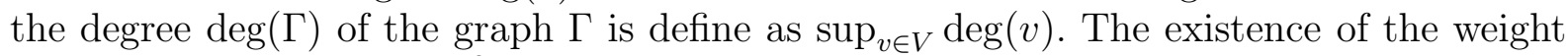
$\mu$ implies that $\operatorname{deg}(\Gamma) \leqslant \delta^{2}$. Indeed, by (2), we have that $\mu(a) \leqslant \delta$ for any $a \in C_{1}$. The equality (11) and the inequality of above implies that $\delta^{-1} \leqslant \mu(a) \leqslant \delta$ for any $a \in C_{1}$. By using (2) again, we obtain that $\delta=\sum_{a \in C_{1}: s(a)=v} \mu(a) \geqslant \operatorname{deg}(v) \delta^{-1}$ for any $v \in V$. Therefore, $\operatorname{deg}(\Gamma) \leqslant \delta^{2}$.

We extend $\mu$ on the set of all paths of $\Gamma$ by putting $\mu\left(a_{1} \cdots a_{n}\right)=\mu\left(a_{1}\right) \cdots \mu\left(a_{n}\right)$ where $a_{1} \cdots a_{n}$ is the concatenation path of $n$ edges $a_{1}, \cdots, a_{n}$. Up to multiplication by a strictly positive real number, there exists a unique map $\mu_{V}: V \longrightarrow \mathbb{R}_{+}^{*}$ such that $\mu(a)=\mu_{V}(t(a)) / \mu_{V}(s(a))$ for any $a \in C_{1}$. Indeed, let us fix a vertex $o \in V$ and put $\mu_{V}(o)=1$ and $\mu_{V}(v)=\mu(a)$ where $a$ is any path such that $s(a)=o$ and $t(a)=v$. Since $\Gamma$ is assumed to be connected, there is always a path from $o$ to $v$. Condition (1) assures that $\mu_{V}$ is well defined. Suppose there is another weight $\nu$ satisfying that $\mu(a)=\nu(t(a)) / \nu(s(a))$ for any $a \in C_{1}$. We can see that $\nu(v)=\mu_{V}(v) \nu(o)$ for any $v \in V$. Hence, $\mu_{V}$ is unique up to a multiplication by a strictly positive real number. This map satisfies that $A_{\Gamma}\left(\mu_{V}\right)=\delta \mu_{V}$, where $A_{\Gamma}$ is the adjacency matrix of the graph $\Gamma$. Following [14, 7], the data of $\left(\Gamma, \mu_{V}\right)$ allows us to define a planar algebra structure on $\mathcal{P}_{\Gamma}=\mathcal{P}=\left(\mathcal{P}_{n}^{ \pm}: n \geqslant 0\right)$. Note that this planar algebra structure only depends on $\Gamma$ and $\mu$. We say that $\mathcal{P}$ is the bipartite graph planar algebra associated to the weighted graph $(\Gamma, \mu)$.

The bipartite graph planar algebra $\mathcal{P}$ is known to satisfy the identity

$$
\tau_{l}\left(e_{a, b}\right)=\delta_{a, b} e_{t(a)} \mu(\bar{a}) \text { and } \tau_{r}\left(e_{a, b}\right)=\delta_{a, b} e_{s(a)} \mu(a)
$$

for any $(a, b) \in S T_{*}^{ \pm}$, where $\delta_{a, b}$ is the Kronecker symbol.

Note that condition (11) on $\mu$ assures the existence of $\mu_{V}$ and implies that $\tau_{l}$ and $\tau_{r}$ are tracial. More precisely, consider the collection of $*$-algebras $\left(\mathcal{P}_{n}^{ \pm}: n \geqslant 0\right)$ and the linear functional $\tau_{l}, \tau_{r}: \mathcal{P}_{n}^{ \pm} \longrightarrow \mathcal{P}_{0}, n \geqslant 0$ defined on the system of matrix units by equation (3). Then $\tau_{l}, \tau_{r}$ are tracial if and only if $\mu(a)=\mu(b)$ for any $(a, b) \in S T_{*}^{ \pm}$. Indeed, suppose that $\tau_{r}$ is tracial. If $(a, b) \in S T_{*}^{ \pm}$, then $\tau_{r}\left(e_{a, b} e_{b, a}\right)=e_{s(a)} \mu(a)=$ $\tau_{r}\left(e_{b, a} e_{a, b}\right)=e_{s(a)} \mu(b)$. Conversely, suppose that $\mu(a)=\mu(b)$ for any $(a, b) \in S T_{*}^{ \pm}$. Consider $n \geqslant 1, x=\sum_{(a, b) \in S T_{n}^{ \pm}} x_{a, b} e_{a, b}, y=\sum_{(a, b) \in S T_{n}^{ \pm}} y_{a, b} e_{a, b}$. We have that $\tau_{r}(x y)=$ $\sum_{(a, b) \in S T_{n}^{ \pm}} x_{a, b} y_{b, a} \mu(a) e_{s(a)}$ and $\tau_{r}(y x)=\sum_{(a, b) \in S T_{n}^{ \pm}} y_{b, a} x_{a, b} \mu(b) e_{s(b)}=\sum_{(a, b) \in S T_{n}^{ \pm}} x_{a, b} y_{b, a} \mu(a) e_{s(a)}=$ $\tau_{r}(x y)$. A similar proof shows that $\tau_{l}$ is also tracial.

1.2. Amenability. All groups that we consider are either countable discrete or locally compact second countable. This implies that any quotient space by a closed subgroup is countable. Recall that a locally compact group $G$ is amenable if and only if for any affine 
action of $G$ on a compact convex subset of a locally convex topological vector space there exists a $G$-fixed point. Eymard defined and studied amenability of inclusion of groups [10]. A closed subgroup $H<G$ is co-amenable if and only if for any affine action of $G$ on a compact convex subset of a locally convex topological vector space that admits a $H$-fixed point there exists a $G$-fixed point. Remark that if $G$ is an amenable group, then any closed subgroup $H<G$ is co-amenable. There are alternative definitions of co-amenability when the subgroup $H<G$ is almost normal, i.e. for any $g \in G$ the group $H \cap g \mathrm{Hg}^{-1}$ has finite index inside $H$ and $g \mathrm{Hg}^{-1}$. It has been proven in [1, Theorem 3.8] that a closed almost normal subgroup $H<G$ is co-amenable if and only if there exists a sequence of positive definite $H$-bi-invariant maps $f_{n}: G \longrightarrow \mathbb{C}, n \geqslant 0$ with support contained in a finite union of right $H$-cosets and that converges pointwise to 1 . We will use later this characterization.

Recall that if $\mathcal{N} \subset \mathcal{M}$ is an inclusion of $\mathrm{II}_{1}$ factors and $L^{2}(\mathcal{M}, \tau)$ is the GNS Hilbert space associated to the unique normal faithful tracial state of $\mathcal{M}$ we say that a $\mathcal{N}$ bimodule $K \subset L^{2}(\mathcal{M}, \tau)$ is bifinite if there exists a finite subset $F \subset L^{2}(\mathcal{M}, \tau)$ such that $K$ is contained in the closure of $\operatorname{Span}(x \cdot \xi ; x \in \mathcal{N}, \xi \in F)$ and in the closure of $\operatorname{Span}(\xi \cdot x: x \in \mathcal{N}, \xi \in F)$. Similarly to the group case, we say that an inclusion of $\mathrm{II}_{1}$ factors $\mathcal{N} \subset \mathcal{M}$ is co-amenable if there exists a sequence of completely positive $\mathcal{N}$ bimodular maps $\phi_{n}: \mathcal{M} \longrightarrow \mathcal{M}$ such that their image is a bifinite $\mathcal{N}$-bimodule and $\lim _{n \rightarrow \infty}\left\|\phi_{n}(x)-x\right\|_{2}=0$ for any $x \in \mathcal{M}$.

Let $\mathcal{Q}$ be a subfactor planar algebra with loop parameter $\delta$. Popa defined amenability for subfactors and $\lambda$-lattices [27]. In planar algebraic terms, $\mathcal{Q}$ is amenable if $\|\Gamma(\mathcal{Q})\|=\delta$, where $\Gamma(\mathcal{Q})$ is the principal graph of $\mathcal{Q}$ deduced from the Bratteli diagram of the tower of finite dimensional semi-simple $*$-algebras $\mathcal{Q}_{0}^{+} \subset \mathcal{Q}_{1}^{+} \subset \cdots$ and where $\|\Gamma(\mathcal{Q})\|$ is the operator norm of the adjacency matrix of $\Gamma$ acting on the $\ell^{2}$-space of the vertices of $\Gamma(\mathcal{Q})$. If $\mathcal{Q}$ is a subfactor planar algebra, then we can associate a sequence of symmetric enveloping inclusions $T_{k} \subset S_{k}, k \geqslant 0$ as constructed in [8]. Those are inclusions of $\mathrm{II}_{1}$ factors. It can be shown that $T_{1} \subset S_{1}$ is isomorphic to the symmetric enveloping inclusion of a subfactor having its subfactor planar algebra isomorphic to $\mathcal{Q}$, see [8, Theorem 3.3]. Popa proved that $\mathcal{Q}$ is amenable if and only if the symmetric enveloping inclusion $T_{1} \subset S_{1}$ is co-amenable [30, Theorem 5.3]. Furthermore, up to a compression and finite index inclusions we have that $T_{1} \subset S_{1}$ is isomorphic to $T_{0} \subset S_{0}$ [6, Lemma 4.3]. Therefore, $\mathcal{Q}$ is amenable if and only if $T_{0} \subset S_{0}$ is co-amenable. We will later use this characterization of amenability of subfactor planar algebras.

1.3. A criterion of amenability for subfactor planar algebras. We provide a criteria of non-amenability for a subfactor planar algebra embedded in a bipartite graph planar algebra. The proof of this criteria is elementary and is interesting by its own.

Theorem 1.1. Let $(\Gamma, \mu, \delta)$ be a weighted graph with a modulus such that $\|\Gamma\|<\delta$. Consider its associated bipartite graph planar algebra $\mathcal{P}$. If $\mathcal{Q}$ is a subfactor planar algebra that embeds inside $\mathcal{P}$, then $\mathcal{Q}$ is non-amenable.

Proof. Let $\Gamma, \mu, \delta, \mathcal{Q}$ be as above. Denote by $\Gamma(\mathcal{Q})$ the principal graph of $\mathcal{Q}$. It is sufficient to show that $\|\Gamma(\mathcal{Q})\| \leqslant\|\Gamma\|$ since $\mathcal{Q}$ and $\mathcal{P}$ have the same modulus $\delta$. Let $\Gamma(\mathcal{Q})_{n}$ be the Bratteli diagram of the inclusion $\mathcal{Q}_{n}^{+} \subset \mathcal{Q}_{n+1}^{+}$for $n \geqslant 0$. We have the equality

$$
\|\Gamma(\mathcal{Q})\|=\lim _{n \rightarrow \infty}\left\|\Gamma(\mathcal{Q})_{n}\right\| .
$$


Let $\Delta_{n}$ be the Bratteli diagram of the inclusion $\mathcal{P}_{n}^{+} \subset \mathcal{P}_{n+1}^{+}$. This graph is equal to the disjoint union of graphs $\Delta_{n}=\bigsqcup_{v \in V^{+}} \Delta_{n}(v)$, where $\Delta_{n}(v)$ is the subgraph of $\Gamma$ with vertices $V_{n}(v)^{ \pm}=\left\{w \in V^{ \pm}: d(v, w) \leqslant n+1\right\}$ and edges the one of $\Gamma$. Since $\Delta_{n}$ is a subgraph of $\Gamma$ we have that

$$
\left\|\Delta_{n}\right\| \leqslant\|\Gamma\|, \text { for any } n \geqslant 0 \text {. }
$$

The planar algebras $\mathcal{Q}$ is contained inside $\mathcal{P}$. Fix $n \geqslant 0$ and consider the following square of inclusions

$$
\begin{array}{cccc}
\mathcal{P}_{n}^{+} & \subset & \mathcal{P}_{n+1}^{+} \\
\cup & & \cup \\
\mathcal{Q}_{n}^{+} & \subset & \mathcal{Q}_{n+1}^{+}
\end{array} .
$$

Consider the normalized tracial operator

$$
t r_{m}: \mathcal{P}_{m}^{+} \longrightarrow \mathcal{P}_{0}^{+}, x \longmapsto \frac{1}{\delta^{m}}\{\text { for any } m \geqslant 0 .
$$

Observe that the restriction of $t r_{n+1}$ to $\mathcal{P}_{n}^{+}$is equal to $t r_{n}$. We equipped $\mathcal{P}_{n}^{+}, \mathcal{Q}_{n}^{+}$, and $\mathcal{Q}_{n+1}^{+}$with the corresponding restrictions of $t r_{n+1}$. Note that

$$
E_{\mathcal{P}}: \mathcal{P}_{n+1}^{+} \longrightarrow \mathcal{P}_{n}^{+}, x \longmapsto \frac{1}{\delta} \stackrel{n}{n} \sqrt[n]{n}
$$

is a faithful conditional expectation satisfying $t r_{n} \circ E_{\mathcal{P}}=t r_{n+1}$. The restriction of $E_{\mathcal{P}}$ to $Q_{n+1}^{+}$is a trace-preserving conditional expectation from $\mathcal{Q}_{n+1}^{+}$onto $\mathcal{Q}_{n}^{+}$. Therefore, the square of inclusion (6) is a commuting square of inclusions with respect to the conditional expectations $E_{\mathcal{P}}$ and $\left.E_{\mathcal{P}}\right|_{\mathcal{Q}_{n+1}^{+}}$. Let $L_{n}$ be the von Neumann algebra generated by $\mathcal{P}_{n}^{+}$and $\mathcal{Q}_{n+1}^{+}$inside $\mathcal{P}_{n+1}^{+}$. Denote by $\Lambda_{n}$ the Bratteli diagram of the inclusion $\mathcal{P}_{n}^{+} \subset L_{n}$. The inclusions $\mathcal{Q}_{n}^{+} \subset \mathcal{Q}_{n+1}^{+} \subset L_{n}$ and $\mathcal{P}_{n}^{+} \subset L_{n}$ form a non-degenerate commuting square. Therefore, $\left\|\Gamma(\mathcal{Q})_{n}\right\|=\left\|\Lambda_{n}\right\|$ by [18, Remark 5.3.5]. This implies that

$$
\left\|\Gamma(\mathcal{Q})_{n}\right\| \leqslant\left\|\Delta_{n}\right\|, \text { for any } n \geqslant 0 .
$$

We obtain the result by combining (4), (5), and (7).

Remark 1.2. The embedding theorem of [16, 21] implies the following. If $\mathcal{Q}$ is a nonamenable subfactor planar algebra, then there exists a bipartite graph planar algebra $\mathcal{P}$ associated to a weighted graph with a modulus $(\Gamma, \mu, \delta)$ such that $\|\Gamma\|<\delta$ and such that $\mathcal{Q}$ embeds inside $\mathcal{P}$. Therefore, a subfactor planar algebra $\mathcal{Q}$ is non-amenable if and only if there exists a weighted graph with a modulus $(\Gamma, \mu, \delta)$ such that $\|\Gamma\|<\delta$ and such that $\mathcal{Q}$ embeds in the bipartite graph planar algebra associated to $(\Gamma, \mu, \delta)$.

\section{Construction of von Neumann algebras}

2.1. Von Neumann algebras associated to a weighted graph with a modulus. We fix a weighted graph $(\Gamma, \mu)$ with modulus $\delta$ and consider the bipartite graph planar algebra $\mathcal{P}=\mathcal{P}_{\Gamma}$. We generalize the construction of [11, 17] and [8] and provide a tower of von Neumann algebras and a family of symmetric enveloping inclusions associated to $\mathcal{P}$. Let $k \geqslant 0$ be a natural number and $\epsilon$ the $\operatorname{sign}+$ is $k$ is even and - if $k$ is odd. For any $n, m \geqslant 0$, let $D_{k}(n, m)$ be a copy of the vector space $\mathcal{P}_{n+m+2 k}^{+}$. 
Consider the direct sum

$$
G r_{k} \mathcal{P} \otimes G r_{k} \mathcal{P}:=\bigoplus_{n, m \geqslant 0} D_{k}(n, m)
$$

that we equipped with the Bacher product:

$$
x y=\sum_{a=0}^{\min (2 n, 2 i)} \sum_{b=0}^{\min (2 m, 2 j)} 2 \mathrm{k}-\underbrace{x}_{\mathrm{b}} y-2 \mathrm{k}
$$

where $x \in D_{k}(n, m), y \in D_{k}(i, j)$, and there are $2 k$ horizontal strands in the middle.

Let $\dagger: G r_{k} \mathcal{P} \otimes G r_{k} \mathcal{P} \longrightarrow G r_{k} \mathcal{P} \otimes G r_{k} \mathcal{P}$ be the anti-linear involution that sends $D_{k}(n, m)$ to itself and satisfies

$$
x^{\dagger}=-\frac{1}{x^{*} \$} \text {, for any } x \in D_{k}(n, m) .
$$

Consider the linear map $E: G r_{k} \mathcal{P} \otimes G r_{k} \mathcal{P} \longrightarrow \mathcal{P}_{0}^{\epsilon}$ which sends $x \in D_{k}(0,0)$ to

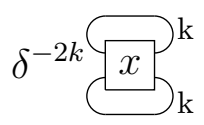

and 0 to any element in $D_{k}(n, m)$ with $(n, m) \neq(0,0)$. The vector space $G r_{k} \mathcal{P} \otimes G r_{k} \mathcal{P}$ endowed with those operation is an associative $*$-algebra with a faithful positive linear map to $\mathcal{P}_{0}^{\epsilon}$. We fix a weight $\mu_{V}: V \longrightarrow \mathbb{R}_{+}^{*}$ that satisfies that $\mu(a)=\mu_{V}(t(a)) / \mu_{V}(s(a))$ for any $a \in C_{1}$. Consider the normal faithful semi-finite weight $\tau_{V}$ satisfying $\tau_{V}\left(e_{v}\right)=$ $\mu_{V}(v)^{2}$ for any $v \in V$. Denote by $H_{k}(n, m)$ the Hilbert space equal to the completion of the pre-Hilbert space $\left\{x \in D_{k}(n, m): \tau_{V} \circ E\left(x x^{\dagger}\right)<\infty\right\}$ equipped with the inner product $\langle x, y\rangle=\tau_{V} \circ E\left(x y^{\dagger}\right)$, for any $n, m \geqslant 0$. Let $H_{k}=\bigoplus_{n, m \geqslant 0} H_{k}(n, m)$ be the direct sum of those Hilbert spaces. Consider $\pi_{k}: G r_{k} \mathcal{P} \otimes G r_{k} \mathcal{P} \longrightarrow \mathcal{L}\left(K_{k}\right)$, the left regular representation of $G r_{k} \mathcal{P} \otimes G r_{k} \mathcal{P}$, where $K_{k}=H_{k} \cap G r_{k} \mathcal{P} \otimes G r_{k} \mathcal{P}$ and $\mathcal{L}\left(K_{k}\right)$ is the algebra of linear maps from $K_{k}$ to $K_{k}$.

Proposition 2.1. For any $x \in G r_{k} \mathcal{P} \otimes G r_{k} \mathcal{P}$, the linear map $\pi_{k}(x)$ defines a bounded operator on $H_{k}$.

Proof. The proof follows the same ideas as [17, Theorem 3.3].

Denote by $S_{k}$ the von Neumann algebra generated by the image of $G r_{k} \mathcal{P} \otimes G r_{k} \mathcal{P}$ inside $B\left(H_{k}\right)$. We continue to denote by $\pi_{k}$ the representation of $S_{k}$ on $H_{k}$. Let $G r_{k} \mathcal{P}$ be the subalgebra of $G r_{k} \mathcal{P} \otimes G r_{k} \mathcal{P}$ generated by $\bigcup_{n \geqslant 0} D_{k}(n, 0)$. We identify the opposite algebra of $G r_{k} \mathcal{P}$ with the subalgebra of $G r_{k} \mathcal{P} \otimes G r_{k} \mathcal{P}$ generated by $\bigcup_{m \geqslant 0} D_{k}(0, m)$ that we denote by $G r_{k} \mathcal{P}^{\text {op }}$. Let $\mathbb{C}\left[V^{\epsilon}\right] \subset \ell^{\infty}\left(V^{\epsilon}\right)$ be the subalgebra generated by the set of projections $\left\{e_{v}, v \in V^{\epsilon}\right\}$. The abelian algebra $\mathbb{C}\left[V^{\epsilon}\right]$ is contained in the center of $G r_{k} \mathcal{P}$ and $G r_{k} \mathcal{P}^{\text {op }}$. Further, the subalgebra of $G r_{k} \mathcal{P} \otimes G r_{k} \mathcal{P}$ generated by $G r_{k} \mathcal{P}$ and $G r_{k} \mathcal{P}^{\text {op }}$ is isomorphic to the tensor product of $G r_{k} \mathcal{P}$ and $G r_{k} \mathcal{P}^{\text {op }}$ over $\mathbb{C}\left[V^{\epsilon}\right]$. We denote this subalgebra by

$$
G r_{k} \mathcal{P} \otimes_{\mathbb{C}\left[V^{\epsilon]}\right.} G r_{k} \mathcal{P}^{\text {op }} \text {. }
$$

Denote by $T_{k}$ and $M_{k}$ the von Neumann subalgebras of $S_{k}$ generated by $G r_{k} \mathcal{P} \otimes_{\mathbb{C}\left[V^{\epsilon}\right]}$ $G r_{k} \mathcal{P}^{\text {op }}$ and $G r_{k} \mathcal{P}$ respectively. The von Neumann algebra generated by $G r_{k} \mathcal{P}^{\text {op }}$ is isomorphic to the opposite von Neumann algebra of $M_{k}$. We denote it by $M_{k}^{\text {op }}$. There exists an inclusion of *-algebras $i_{k}: G r_{k} \mathcal{P} \otimes_{\mathbb{C}\left[V^{\epsilon}\right]} G r_{k} \mathcal{P}^{\text {op }} \longrightarrow G r_{k+1} \mathcal{P} \otimes_{\mathbb{C}\left[V^{\varepsilon}\right]} G r_{k+1} \mathcal{P}^{\text {op }}$ which 
consists of adding two horizontal lines in the middle and dividing by $\delta^{2}$, where $\varepsilon$ is + if $k$ is odd and - if $k$ is even. This inclusion induces unital embeddings from $T_{k}$ into $T_{k+1}$, $M_{k}$ into $M_{k+1}$, and $M_{k}^{\text {op }}$ into $M_{k+1}^{\text {op }}$.

2.2. Properties associated to those von Neumann algebras. For any $n, m \geqslant 0$, denote by $j_{k}(n, m)$ the canonical embedding of $\mathcal{P}_{n+m+2 k}^{+}$into $D_{k}(n, m)$ viewed as a subspace of $S_{k}$. For any vertex $v \in V^{\epsilon}$, consider the projection

$$
p_{v}=\frac{\frac{\mathrm{k}}{\frac{e_{v}}{\mathrm{k}}}}{e} j_{k}(0,0)\left(e_{v}\right) \in S_{k} .
$$

This projection belongs to $M_{k} \cap M_{k}^{\text {op }}$ and commutes with $T_{k}$. Denote by $T_{k}(v), M_{k}(v), M_{k}(v)^{\text {op }}$ the corners $T_{k} p_{v}, M_{k} p_{v}$, and $M_{k}^{\mathrm{op}} p_{v}$ respectively.

Proposition 2.2. The following assertions are true.

(1) The von Neumann algebra $T_{k}(v)$ is isomorphic to the tensor product $M_{k}(v) \bar{\otimes} M_{k}(v)^{o p}$ and $M_{k}(v)$ is a $I I_{1}$ factor, for any $v \in V^{\epsilon}$.

(2) The corner $p_{v} S_{k} p_{v}$ is equal to $T_{k}(v)$ for any $v \in V^{\epsilon}$.

(3) The relative commutant $T_{k}^{\prime} \cap S_{k}$ is equal to the center $Z\left(T_{k}\right)$ of $T_{k}$ and is isomorphic to $\mathcal{P}_{0}^{\epsilon}$. The set of minimal central projections of $T_{k}$ is $\left\{p_{v}: v \in V^{\epsilon}\right\}$.

(4) The von Neumann algebra $S_{k}$ is a type II factor. It is a finite factor if and only if the graph $\Gamma$ is finite. Up to a scalar, the unique normal faithful semi-finite tracial weight of $S_{k}$ is defined as $\operatorname{Tr}(x)=\sum_{v \in V^{\epsilon}}\left\langle\pi_{k}(x) p_{v}, p_{v}\right\rangle$ for any positive operator $x \in S_{k}$. Moreover, $\operatorname{Tr}(y)=\tau_{V} \circ E(y)$ for any positive operator $y \in G r_{k} \mathcal{P} \otimes G r_{k} \mathcal{P}$.

Proof. We assume that $k=0$ and drop the subscript $k$. The general case can easily be deduced.

Proof of (1). Consider a vertex $v \in V^{+}$. It is obvious that $M(v)$ and $M(v)^{\text {op }}$ commute and generate the von Neumann algebra $T(v)$. Following [17, Corollary 4.12], we obtain that $M(v)$ is a $\mathrm{II}_{1}$ factor. Let $\tau$ be the normal faithful tracial state of $M(v)$. Consider the map $E: G r \mathcal{P} \otimes G r \mathcal{P} \longrightarrow \mathcal{P}_{0}^{+}$defined in Section 2.1 and its restriction $\varphi$ to $T(v) \cap G r \mathcal{P} \otimes G r \mathcal{P}$ that has values in $\mathbb{C} p_{v} \simeq \mathbb{C}$. Observe that $\varphi\left(a_{1} b_{1}^{\text {op }} \cdots a_{n} b_{n}^{\text {op }}\right)=$ $\tau\left(a_{1} \cdots a_{n}\right) \tau\left(b_{1} \cdots b_{n}\right)$ for any $a_{1}, \cdots, a_{n} \in p_{v} G r \mathcal{P}$ and $b_{1}^{\text {op }}, \cdots, b_{n}^{\text {op }} \in p_{v} G r \mathcal{P}^{\text {op }}$. This implies that $T(v)$ is isomorphic to $M(v) \bar{\otimes} M(v)^{\mathrm{op}}$.

Proof of (2). Consider a loop $l$ of length $2 n+2 m$ that starts at an even vertex $v$. It defines a partial isometry $e_{a, b} \in \mathcal{P}_{n+m}^{+} \subset B\left(C_{n+m}^{+}\right)$, where $a$ is the path equal to the first half of the loop and $b$ the opposite path equal to the second half of the loop. Consider the corresponding element $x_{l}:=j(n, m)\left(e_{a, b}\right) \in S$. If $p_{v} x_{l} p_{v}=x_{l}$, then the $2 \mathrm{n}$-th vertex in the loop $l$ is also equal to $v$. This implies that $l$ is the concatenation of two loops $l_{1}$ and $l_{2}$ where $l_{1}$ is the truncation of $l$ for the $2 \mathrm{n}$-th first edges and $l_{2}$ is the rest of the loop $l$. Let $y_{i}=e_{a_{i}, b_{i}}$ be the partial isometry of $\mathcal{P}_{n}^{+}$if $i=1$ and $\mathcal{P}_{m}^{+}$if $i=2$ such that the concatenation of the path $a_{i}$ and the opposite of $b_{i}$ is equal to the loop $l_{i}$ for $i=1,2$. We have that $x_{l}=x_{l_{1}} \otimes x_{l_{2}}$, where $x_{l_{1}}=j(n, 0)\left(e_{a_{1}, b_{1}}\right)$ and $x_{l_{2}}=j(0, m)\left(e_{a_{2}, b_{2}}\right)$,

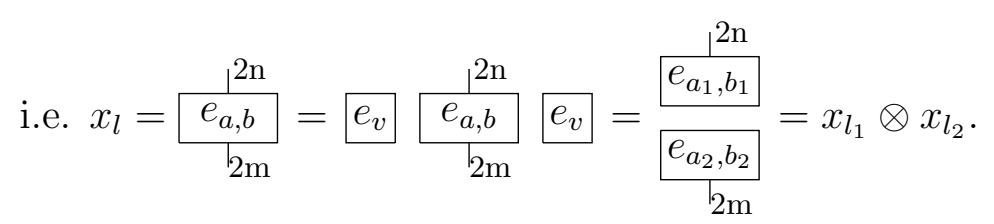


Therefore, $p_{v} D(n, m) p_{v}$ is contained in $T(v)$ for any $v \in V^{+}$and $n, m \geqslant 0$. By density, we obtain that $p_{v} S p_{v}=T(v)$.

Proof of (3). Consider $x$ in the relative commutant $T^{\prime} \cap S$. For any $v, w \in V^{+}$we have that $p_{v} x p_{w}=p_{v} p_{w} x$. Therefore, $x$ is in $T$ by (2). We obtain that $x$ is in the center of $T$. We conclude by using (1).

Proof of (4). Consider an element $x$ in the center of $S$. By (3) this element belongs to the center of $T$. Hence, there exists a bounded map $f: V^{+} \longrightarrow \mathbb{C}$ such that $x=$ $\sum_{v \in V^{+}} f(v) p_{v}$. Consider two vertices $v$ and $w$. There exists a path $c$ of length $n$ in $\Gamma$ that starts at $v$ and ends at $w$ since the graph is connected. Consider the corresponding nonzero projection $e_{c, c} \in \mathcal{P}_{n}^{+}$and its image $y:=j(n, n)\left(e_{c, c}\right)$ in $S$. Observe that $y x=f(w) y$ and $x y=f(v) y$. Therefore, $f$ is constant and the center of $S$ is trivial. We deduce that $S$ is a factor. We have that $p_{v} S p_{v}$ is a $\mathrm{II}_{1}$ factor by (1) and (2). Therefore, $S$ is a type II factor. Consider the weight $\operatorname{Tr}$ of $S$ defined by $\operatorname{Tr}(x)=\sum_{v \in V^{+}}\left\langle\pi(x) p_{v}, p_{v}\right\rangle$, where $\langle\cdot, \cdot\rangle$ is the inner product of $H$. It is clear that this weight is normal, semi-finite, faithful, and sends $p_{v}$ to $\mu_{V}(v)^{2}$ for any $v \in V^{+}$. Consider a positive operator $y \in G r \mathcal{P} \otimes G r \mathcal{P}$. Observe that

$$
\begin{aligned}
\sum_{v \in V^{+}}\left\langle\pi(y) p_{v}, p_{v}\right\rangle & =\sum_{v \in V^{+}} \tau_{V} \circ E\left(y p_{v} p_{v}^{*}\right)=\sum_{v \in V^{+}} \tau_{V} \circ E\left(y p_{v}\right) \\
& =\tau_{V} \circ E\left(y \sum_{v \in V^{+}} p_{v}\right)=\tau_{V} \circ E(y) .
\end{aligned}
$$

Consider two loops $l, k$ of length $2 n+2 m$ and $2 t+2 s$ in $\Gamma$ that start at $v \in V^{+}$ and $w \in V^{+}$respectively. Consider $x_{l}$ and $x_{k}$ the corresponding elements of $S$ given by the inclusion maps $j(n, m)$ and $j(t, s)$. The identity (3) of Section 1 implies that $\operatorname{Tr}\left(x_{l} x_{k}\right)=\delta_{n, t} \delta_{m, s} \mu_{V}(w) \mu_{V}(v)=\operatorname{Tr}\left(x_{k} x_{l}\right)$, where $\delta_{n, t}$ is the Kronecker symbol. We obtain by density that $\operatorname{Tr}$ is tracial. By uniqueness of a normal faithful tracial weight on a type II factor, we have that $S$ is a finite von Neumann algebra if and only if $\operatorname{Tr}(1)<\infty$. Suppose that $\Gamma$ is finite. Then $\operatorname{Tr}(1)=\sum_{v \in V^{+}} \operatorname{Tr}\left(p_{v}\right)=\sup _{v \in V^{+}} \operatorname{Tr}\left(p_{v}\right)\left|V^{+}\right|<\infty$, where $\left|V^{+}\right|$is the cardinal of $V^{+}$. Hence, $S$ is a $\mathrm{II}_{1}$ factor. Suppose that $\Gamma$ is infinite. Assume that $\left\{\mu_{V}(v): v \in V^{+}\right\}$is unbounded. Then, $\operatorname{Tr}(1) \geqslant \sup _{v \in V^{+}} \mu_{V}(v)^{2}=\infty$. Assume that $\left\{\mu_{V}(v): v \in V^{+}\right\}$is bounded by $C>0$. Since $\delta^{-1} \leqslant \mu(a) \leqslant \delta$ and $\mu(a)=$ $\mu_{V}(t(a)) / \mu_{V}(s(a))$ for any $a \in C_{1}$, we obtain that $\left\{\mu_{V}(v): v \in V^{+}\right\}$is bounded below by a constant $D>0$. Then, $\operatorname{Tr}(1)=\sum_{v \in V^{+}} \operatorname{Tr}\left(p_{v}\right)=\sum_{v \in V^{+}} \mu_{V}(v)^{2} \geqslant D\left|V^{+}\right|=\infty$. Therefore, $S$ is a $\mathrm{II}_{\infty}$ factor if $\Gamma$ is infinite.

We denote by $L^{2}\left(S_{k}, T r\right)$ the GNS Hilbert space associated to $S_{k}$ and $\operatorname{Tr}$ and identify it with the Hilbert space $H_{k}$.

2.3. Planar algebras contained in a bipartite graph planar algebra. Consider a planar algebra $\mathcal{Q}$ which embeds in the bipartite graph planar algebra $\mathcal{P}$. We identify $\mathcal{Q}$ and its image in $\mathcal{P}$. Consider the *-algebras $G r_{k} \mathcal{Q}, G r_{k} \mathcal{Q}^{\text {op }}$, and $G r_{k} \mathcal{Q} \otimes G r_{k} \mathcal{Q}$ that we identify with subalgebras of $G r_{k} \mathcal{P}, G r_{k} \mathcal{P}^{\text {op }}$, and $G r_{k} \mathcal{P} \otimes G r_{k} \mathcal{P}$ respectively. Denote by $S_{k}(\mathcal{Q})$, $M_{k}(\mathcal{Q})$, and $M_{k}(\mathcal{Q})^{\text {op }}$ the von Neumann subalgebras of $S_{k}$ generated by $G r_{k} \mathcal{Q} \otimes G r_{k} \mathcal{Q}$, $G r_{k} \mathcal{Q}$, and $G r_{k} \mathcal{Q}^{\text {op }}$ respectively. Let $T_{k}(\mathcal{Q})=M_{k}(\mathcal{Q}) \vee M_{k}(\mathcal{Q})^{\text {op }}$ be the von Neumann subalgebra of $S_{k}$ generated by $M_{k}(\mathcal{Q})$ and $M_{k}(\mathcal{Q})^{\text {op }}$. 
Proposition 2.3. Let $\mathcal{J}$ be the Temperley-Lieb-Jones planar algebra included in $\mathcal{P}$, i.e. the planar subalgebra of $\mathcal{P}$ generated by tangles without inner discs. Then, $S_{k}(\mathcal{J}) \subset S_{k}$ is an irreducible subfactor. Moreover, $T_{k}(\mathcal{J})^{\prime} \cap S_{k}=Z\left(T_{k}\right)$.

Proof. We assume that $k=0$ and drop the subscript $k$. The general case can easily be deduced. Consider the element $\cup \in D(1,0)$ and $\cap \in D(0,1)$. Denote by $C$ and $C^{\mathrm{op}}$ the abelian von Neumann algebras generated by $\cup$ and $\cap$ respectively. Let $A=C \vee C^{\text {op }}$ be the abelian von Neumann algebra generated by $C$ and $C^{\mathrm{op}}$. Consider the von Neumann algebra $B=A \vee Z(T)$ generated by $A$ and the set of projections $\left\{p_{v}: v \in V^{+}\right\}$. We claim that the relative commutant $S \cap A^{\prime}$ is contained in $B$. Consider the following subspaces of $S$ :

$$
\begin{gathered}
W^{t, n}(v)=\left\{x \in D(n, 0) p_{v}: \stackrel{2 \mathrm{n}-2 \mid}{x}=\int_{x}^{\mid 2 \mathrm{n}-2}=0\right\}, \\
W_{b, m}(v)=\left\{x \in D(0, m) p_{v}: \underset{2 \mathrm{~m}-2]}{x}=\underset{x_{2 \mathrm{~m}-2}}{x^{2}}=0\right\}, \text { and }
\end{gathered}
$$

$W_{b, m}^{t, n}(v, w)$ the space of $x \in p_{w} D(n, m) p_{v}$ such that

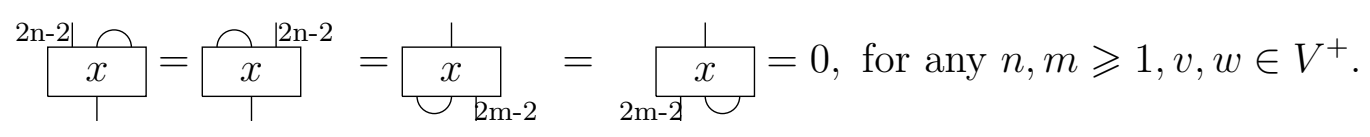

Observe that all those spaces are contained in $L^{2}(S, T r)$ and are pairwise orthogonal. Denote by $W^{t}(v)=\bigoplus_{n \geqslant 1} W^{t, n}(v), W_{b}(v)=\bigoplus_{m \geqslant 1} W_{b, m}(v)$, and $W_{b}^{t}(v, w)=\bigoplus_{n, m \geqslant 1} W_{b, m}^{t, n}(v, w)$ the direct sum of those spaces inside $L^{2}(S, T r)$ for any $v, w \in V^{+}$. Let $H^{t}(v), H_{b}(v)$, and $H_{b}^{t}(v, w)$ be the $A$-bimodules generated by $W^{t}(v), W_{b}(v)$, and $W_{b}^{t}(v, w)$ respectively for any $v, w \in V^{+}$. Consider the $A$-bimodule generated by $D(0,0)$ inside $L^{2}(S, T r)$. It is isomorphic to $L^{2}(A) \otimes \ell^{2}\left(V^{+}\right)$as a $A$-bimodule. We identify those two bimodules. A similar proof to [17, Theorem 4.9] shows that we have the following decomposition into A-bimodules:

$$
L^{2}(S, T r)=\left(L^{2}(A) \otimes \ell^{2}\left(V^{+}\right)\right) \oplus \bigoplus_{v \in V^{+}} H^{t}(v) \oplus \bigoplus_{v \in V^{+}} H_{b}(v) \oplus \bigoplus_{v, w \in V^{+}} H_{b}^{t}(v, w)
$$

Consider $x \in S \cap A^{\prime}$ and two different even vertices $v \neq w$. Observe that $p_{v}$ and $p_{w}$ commute with $A$. Furthermore, $p_{v} x p_{w}$ is in $L^{2}(S, T r)$. Hence, $p_{v} x p_{w}$ is in $L^{2}(S, T r) \cap A^{\prime}$. The equality (8) implies that $p_{v} x p_{w}$ is in $H_{b}^{t}(v, w)$. Therefore, $p_{v} x p_{w}$ is a $A$-central vector of $H_{b}^{t}(v, w)$. Following [17, Theorem 4.9], we can prove that the $A$-bimodule $H_{b}^{t}(v, w)$ is isomorphic to $L^{2}(A) \otimes W_{b}^{t}(v, w) \otimes L^{2}(A)$. Therefore, it is isomorphic to a direct sum of the coarse $A$-bimodule $L^{2}(A) \otimes L^{2}(A)$. Thus, $H_{b}^{t}(v, w)$ does not admit any nonzero $A$-central vectors. Therefore, $p_{v} x p_{w}=0$ for any $v \neq w$. The element $p_{v} x p_{v}$ is a $A$-central vector of $L^{2}(S, T r)$. The equality (8) implies that $p_{v} x p_{v} \in L^{2}\left(A p_{v}\right) \oplus H^{t}(v) \oplus H_{b}(v) \oplus H_{b}^{t}(v, v)$. By the previous argument we have that the orthogonal component of $p_{v} x p_{v}$ inside $H_{b}^{t}(v, v)$ is equal to 0 . The von Neumann algebra $A$ is isomorphic to $C \bar{\otimes} C^{\text {op }}$. Let $\xi$ be the orthogonal component of $p_{v} x p_{v}$ in $H^{t}(v)$. It is a $C$-central vector. We can prove that the $A$-bimodule $H^{t}(v)$ is isomorphic to $\left(L^{2}(C) \otimes W^{t}(v) \otimes L^{2}(C)\right) \otimes L^{2}\left(C^{\mathrm{op}}\right)$. In particular, the $C$-bimodule $H^{t}(v)$ is isomorphic to a direct sum of the coarse bimodule $L^{2}(C) \otimes L^{2}(C)$. Therefore, $\xi=0$. A similar argument shows that the orthogonal component of $p_{v} x p_{v}$ in $H_{b}(v)$ is equal to 0 . We obtain that $p_{v} x p_{v}$ is in the $A$-bimodule generated by $p_{v}$. Therefore, $x$ belongs to $B$. This proves the claim. 
Consider $x \in S \cap T(\mathcal{J})^{\prime}$. Since $A$ is contained in $T(\mathcal{J})$, we have that $x \in A^{\prime} \cap S \subset B$. The element $p_{v} x$ is in $L^{2}\left(A p_{v}\right)$ for any $v \in V^{+}$. It commutes with the two elements

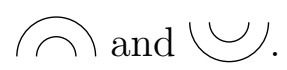

A similar argument to [17, Corollary 4.11] shows that $p_{v} x \in \mathbb{C} p_{v}$ for any $v \in V^{+}$. Since $x=\sum_{v \in V^{+}} p_{v} x$, we obtain that $x \in Z(T)$. In particular, $T(\mathcal{J})^{\prime} \cap S=Z(T)$.

Suppose that $x \in S \cap S(\mathcal{J})^{\prime}$. By the argument of above, we have that $x \in Z(T)$. Hence, there exists a bounded function $f: V^{+} \longrightarrow \mathbb{C}$ such that $x=\sum_{v \in V^{+}} f(v) p_{v}$. Consider the element $\| \in D(1,1) \subset S(\mathcal{J})$ which commutes with $x$. Observe that $p_{v} \| p_{w} \neq 0$ if $v=w$ or if $d(v, w)=2$, where $d$ is the length metric of the graph $\Gamma$. Therefore, $p_{v} x\left\|p_{w}=f(v) p_{v}\right\| p_{w}=p_{v}\left\|x p_{w}=f(w) p_{v}\right\| p_{w}$ for any $v, w \in V^{+}$such that $d(v, w)=2$. This implies that $f(v)=f(w)$ if $d(v, w)=2$. Since $\Gamma$ is connected, we obtain that $f$ is constant. Therefore, $x \in \mathbb{C} 1$.

Part of the following proposition can be deduced from [8]. We provide a full proof for the convenience of the reader.

Proposition 2.4. Consider a subfactor planar algebra $\mathcal{Q}$ which is contained in $\mathcal{P}$. Then $S_{k}(\mathcal{Q})$ is a II $I_{1}$ factor. If $v \in V^{\epsilon}$, then the map $\operatorname{tr}: S_{k}(\mathcal{Q}) \longrightarrow \mathbb{C}, x \longmapsto \mu_{V}(v)^{-2}\left\langle\pi_{k}(x) p_{v}, p_{v}\right\rangle$ is the unique normal faithful tracial state of $S_{k}(\mathcal{Q})$.

Proof. Let $\mathcal{J}$ be the Temperley-Lieb-Jones planar algebra with modulus $\delta$. We have a chain of inclusions $\mathcal{J} \subset \mathcal{Q} \subset \mathcal{P}$. This implies that we have the chain of inclusions $S_{k}(\mathcal{J}) \subset S_{k}(\mathcal{Q}) \subset S_{k}(\mathcal{P})=S_{k}$. We obtain that $S_{k}(\mathcal{Q})$ is a factor since $S_{k}(\mathcal{J}) \subset S_{k}(\mathcal{P})$ is an irreducible subfactor by Proposition 2.3 .

For any vertex $v \in V^{\epsilon}$ we consider the linear functional

$$
\operatorname{tr}_{v}: S_{k}(\mathcal{Q}) \longrightarrow \mathbb{C}, x \longmapsto \mu_{V}(v)^{-2}\left\langle\pi_{k}(x) p_{v}, p_{v}\right\rangle .
$$

The linear function $t r_{v}$ is a normal state since it is a vector state. Denote by $B$ the *-algebra $G r_{k} \mathcal{Q} \otimes G r_{k} \mathcal{Q}$.

Since $\mathcal{Q}$ is a subfactor planar algebra, we have that $\mathcal{Q}^{\epsilon}$ is one dimensional and can be identified to the space of constant functions of $\mathcal{P}^{\epsilon} \simeq \ell^{\infty}\left(V^{\epsilon}\right)$. Consider $b \in B$ and the element $E(b) \in \mathcal{Q}^{\epsilon}$, where $E: G r_{k} \mathcal{Q} \otimes G r_{k} \mathcal{Q} \longrightarrow \mathcal{P}_{0}^{\epsilon}$ is the map defined in Section 2.1. Observe that $\operatorname{tr}_{v}(b)$ is the value of $E(b)$ at the vertex $v$. Since $E(b)$ is constant, we have that $t r_{v}(b)=t r_{w}(b)$ for any $v, w \in V^{\epsilon}$. By density, we obtain that $t r_{v}=t r_{w}$ for any $v, w \in V^{\epsilon}$.

We fix $v \in V^{\epsilon}$. Let us show that $t r:=t r_{v}$ is tracial. By density, it is sufficient to show that $\operatorname{tr}(a b)=\operatorname{tr}(b a)$ for any $a, b \in B$. Consider $a, b \in B$ and denote by $a_{n, m}$ and $b_{n, m}$ their $(n, m)$-component in $D_{k}(n, m), n, m \geqslant 0$. Recall that $\operatorname{Tr}$ is the weight of $S_{k}$ defined in Proposition 2.2,4. Observe that

$$
\begin{aligned}
\operatorname{tr}(a b) & =\sum_{n, m, i, j \geqslant 0} \operatorname{tr}\left(a_{n, m} b_{i, j}\right)=\sum_{n, m, i, j \geqslant 0} \mu_{V}(v)^{-2} \operatorname{Tr}\left(a_{n, m} b_{i, j} p_{v}\right) \\
& =\sum_{n, m \geqslant 0} \mu_{V}(v)^{-2} \operatorname{Tr}\left(a_{n, m} b_{n, m} p_{v}\right) \text { since } a_{n, m} \perp b_{i, j} p_{v} \text { in } H_{k} \text { if }(n, m) \neq(i, j) \\
& =\sum_{n, m \geqslant 0} \operatorname{tr}\left(a_{n, m} b_{n, m}\right) .
\end{aligned}
$$


Hence, it is sufficient to show that $\operatorname{tr}(a b)=\operatorname{tr}(b a)$ for $a, b \in D_{k}(n, m), n, m \geqslant 0$. We fix $n, m \geqslant 0, a, b \in D_{k}(n, m)$, and $x, y \in \mathcal{Q}_{2 k+n+m}^{+}$such that $j_{k}(n, m)(x)=a, j_{k}(n, m)(y)=b$. Since $\mathcal{Q}$ is spherical, we have that $\operatorname{tr}(a b)=\tau_{l}(x y)$, where $x y$ is the product of $x$ and $y$ in the planar algebra $\mathcal{Q}$ and where $\tau_{l}$ is the left trace of $\mathcal{Q}$. By traciality of $\tau_{l}$, we obtain that $\tau_{l}(x y)=\tau_{l}(y x)=\operatorname{tr}(b a)$. Therefore, $\operatorname{tr}$ is a a normal tracial state of the factor $S_{k}(\mathcal{Q})$. Since $S_{k}(\mathcal{Q})$ is infinite dimensional, we obtain that $S_{k}(\mathcal{Q})$ is a $\mathrm{II}_{1}$ factor and its unique normal faithful tracial state is $t r$.

Proposition 2.5. Consider a subfactor planar algebra $\mathcal{Q}$ which is contained in $\mathcal{P}$. The tower of von Neumann algebras $M_{0}(\mathcal{Q}) \subset M_{1}(\mathcal{Q}) \subset M_{2}(\mathcal{Q}) \subset \cdots$ is isomorphic to the tower constructed in [11]. The inclusion $T_{k}(\mathcal{Q}) \subset S_{k}(\mathcal{Q})$ is isomorphic to the $k$-th symmetric enveloping inclusion constructed in [8]. In particular, the subfactor planar algebra of $M_{0}(\mathcal{Q}) \subset M_{1}(\mathcal{Q})$ is isomorphic to $\mathcal{Q}$ and the symmetric enveloping inclusion associated to $M_{k}(\mathcal{Q}) \subset M_{k+1}(\mathcal{Q})$ is isomorphic to $T_{k+1}(\mathcal{Q}) \subset S_{k+1}(\mathcal{Q})$.

Proof. Let $\mathcal{Q}$ be a subfactor planar algebra contained in $\mathcal{P}$. We put $B=G r_{k} \mathcal{Q} \otimes G r_{k} \mathcal{Q}$. In [8], to $\mathcal{Q}$ is associated a tracial $*$-algebra $(V, \wedge, \dagger, T r)$ where $\wedge$ is the multiplication, $\dagger$ the anti-linear involution, and $\operatorname{Tr}$ a tracial linear functional. They also consider another tracial $*$-algebra $\left(W, \star, \dagger, T r^{\prime}\right)$ where $W$ is a copy of $V$ as a vector space. They provide a map $X: V \longrightarrow W$ which is an isomorphism of tracial $*$-algebras and consider a projection $p_{k,+} \in V$ that satisfies that $X\left(p_{k,+}\right)=p_{k,+}$. Observe that the vector spaces $p_{k,+} W p_{k,+}$ and $B$ are equal. Moreover, the $*$-structures of $\left(p_{k,+} W p_{k,+}, \star, \dagger\right)$ and $(B, \cdot, \dagger)$ are defined by the same planar tangles. If we identify $\mathcal{Q}_{0}^{\epsilon}$ with the complex numbers inside $\mathcal{P}_{0}^{\epsilon}$, then we have that our trace $t r: B \longrightarrow \mathbb{C}$ considered in Proposition 2.4 is equal to the restriction to $B$ of the map $E: G r_{k} \mathcal{P} \otimes G r_{k} \mathcal{P} \longrightarrow \mathcal{P}_{0}^{\epsilon}$ defined in Section 2.1. Observe that by definition and the fact that $\mathcal{Q}$ is spherical, we have that $\left.\delta^{-2 k} \operatorname{Tr}^{\prime}\right|_{p_{k,+} W p_{k,+}}$ and $\left.E\right|_{B}$ are the exact same maps. Therefore, the tracial $*$-algebras $\left(p_{k,+} W p_{k,+}, \star, \dagger, T r^{\prime}\right)$ and $(B, \cdot, \dagger, t r)$ are isomorphic. Thus $(B, \cdot, \dagger, t r)$ is isomorphic to $\left(p_{k,+} V p_{k,+}, \wedge, \dagger, T r\right)$. It is proved in [8] that $V$ acts by bounded operators on the GNS Hilbert space $L^{2}(V, T r)$. This implies that the restriction of $\delta^{-2 k} \operatorname{Tr}$ to $p_{k,+} V p_{k,+}$ that they denote by $\tau_{k} \otimes \tau_{k}$ is a faithful tracial state. Let $L_{k}$ be the GNS completion of $p_{k,+} V p_{k,+}$ with respect to $\tau_{k} \otimes \tau_{k}$ (which is denoted by $M_{k} \otimes M_{k}$ in [8].). It is proved that $L_{k}$ is a $\mathrm{II}_{1}$ factor with unique normal faithful tracial state $\tau_{k} \otimes \tau_{k}$.

Consider the von Neumann algebra $S_{k}(\mathcal{Q})$ which is the bicommutant of $B$ inside $S_{k}$. The von Neumann algebra $S_{k}(\mathcal{Q})$ is a $\mathrm{II}_{1}$ factor and its unique normal faithful tracial state is $t r$. Therefore, $L_{k}$ and $S_{k}(\mathcal{Q})$ are both $\mathrm{II}_{1}$ factors which contain a weakly dense *-subalgebra $X^{-1}(B)$ and $B$ respectively. Moreover, $\tau_{k} \otimes \tau_{k} \circ X^{-1}(b)=\operatorname{tr}(b)$ for any $b \in B$. This implies that there exists an isomorphism $\Phi: L_{k} \longrightarrow S_{k}(\mathcal{Q})$ such that $\Phi\left(X^{-1}(b)\right)=b$ for any $b \in B$. Let $F_{k}$ be the von Neumann algebra generated by $G r_{k} \mathcal{Q}$ and $G r_{k} \mathcal{Q}^{\text {op }}$ inside $L_{k}$. It is easy to see that $X^{-1}$ sends $G r_{k} \mathcal{Q}$ and $G r_{k} \mathcal{Q}^{\text {op }}$ to itself. This implies that $\Phi\left(F_{k}\right)=T_{k}(\mathcal{Q})$. Therefore, $T_{k}(\mathcal{Q}) \subset S_{k}(\mathcal{Q})$ is isomorphic to the $\mathrm{k}$-th symmetric enveloping inclusion constructed in 8 .

A similar proof shows that the tower of von Neumann algebras $M_{0}(\mathcal{Q}) \subset M_{1}(\mathcal{Q}) \subset$ $M_{2}(\mathcal{Q}) \subset \cdots$ is isomorphic to the tower constructed in [11]. The rest of the proposition follows from [11, Theorem 8] and [8, Theorem 3.3].

Definition 2.6. If $\mathcal{P}$ is a bipartite graph planar algebra or a subfactor planar algebra we say that $T_{0} \subset S_{0}$ is the symmetric enveloping inclusion associated to $\mathcal{P}$ and denote it by $T \subset S$. 


\section{Fixed POINT PLANAR ALGEBRAS}

\subsection{Actions of the automorphism group of the bipartite graph planar algebra.} Let $(\Gamma, \mu)$ be a weighted graph with modulus $\delta>0$. Consider the bipartite graph planar algebra $\mathcal{P}$. An automorphism of $\mathcal{P}$ is a sequence of maps $a=\left(a_{n}^{ \pm}: n \geqslant 0\right)$ such that $a_{n}^{ \pm}$ is an automorphism of the von Neumann algebra $\mathcal{P}_{n}^{ \pm}$and such that $a$ commutes with the action of the planar tangles. We denote by $\operatorname{Aut}(\mathcal{P})$ the automorphism group of $\mathcal{P}$.

Let $\operatorname{Aut}(\Gamma)$ be the group of permutations of the vertices that conserve the number of edges connecting pairs of vertices and send even vertices to even vertices. We label each n-fold multiple edges by $\{1, \cdots, n\}$ and extend each element of $\operatorname{Aut}(\Gamma)$ by a permutation of the edges that preserves the labeling. Let $\operatorname{Aut}(\Gamma, \mu)$ be the subgroup of $g \in \operatorname{Aut}(\Gamma)$ such that $\mu(g a)=\mu(a)$ for any edge $a$. We extend the action of $\operatorname{Aut}(\Gamma, \mu)$ to an action on all paths $C_{*}$ of $\Gamma$ that we denote as follows: $\operatorname{Aut}(\Gamma, \mu) \times C_{*} \longrightarrow C_{*},(g, a) \mapsto g a$. Burstein proved that the following map $\gamma: \operatorname{Aut}(\Gamma, \mu) \times \mathcal{P} \longrightarrow \mathcal{P},\left(g, e_{a, b}\right) \mapsto e_{g a, g b}$ defines an embedding of $\operatorname{Aut}(\Gamma, \mu)$ into $\operatorname{Aut}(\mathcal{P})[7]$.

Consider the von Neumann algebra $\mathcal{P}_{1}^{+}$and its unitary group $L=U\left(\mathcal{P}_{1}^{+}\right)$. We recall an argument due to Burstein that explains how the group $L$ embeds in $\operatorname{Aut}(\mathcal{P})$. Consider the map $a \in C_{*} \mapsto \bar{a}$ that reverses the orientation of a path. It induces an injective *morphisms Rev $: \mathcal{P}_{1}^{ \pm} \longrightarrow \mathcal{P}_{1}^{\mp}, e_{a, b} \mapsto e_{\bar{a}, \bar{b}}$. We identify $\mathcal{P}_{n}^{ \pm}$with its image in $\mathcal{P}_{n+1}^{+}$and $\mathcal{P}_{n}^{-}$ with its image in $\mathcal{P}_{n+1}^{+}$. Consider the collection of shift operators $s h: \mathcal{P}_{n}^{ \pm} \longrightarrow \mathcal{P}_{n+2}^{ \pm}, e_{a, b} \mapsto$ $\sum_{c \in C_{2}^{ \pm}} e_{c a, c b}, n \geqslant 0$. If $x \in \mathcal{P}_{1}^{+}$, we consider the element $x_{1}^{+}=x \in \mathcal{P}_{1}^{+}, x_{2}^{+}=x \operatorname{Rev}(x) \in$ $\mathcal{P}_{2}^{+}, x_{2 n+1}^{+}=x_{2 n}^{+} \operatorname{sh}^{n}(x) \in \mathcal{P}_{2 n+1}^{+}, x_{2 n+2}^{+}=x_{2 n+1}^{+} s h^{n} \circ \operatorname{Rev}(x) \in \mathcal{P}_{2 n+2}^{+}$and $x_{1}^{-}=\operatorname{Rev}(x) \in$ $\mathcal{P}_{1}^{-}, x_{2}^{-}=x_{1}^{-} \operatorname{sh}(x) \in \mathcal{P}_{2}^{-}, x_{2 n+1}^{-}=x_{2 n}^{-} s^{n}\left(x_{1}^{-}\right) \in \mathcal{P}_{2 n+1}^{-}, x_{2 n+2}^{-}=x_{2 n+1}^{-} \operatorname{sh}^{n+1}(x) \in \mathcal{P}_{2 n+2}^{-}$ for any $n \geqslant 1$. Consider $u \in L$, we have that $u_{n}^{ \pm}$is a unitary of $\mathcal{P}_{n}^{ \pm}$for any $n \geqslant 1$. We put $u_{0}^{ \pm}=1$ and consider the collection of automorphisms $\left(A d\left(u_{n}^{ \pm}\right): n \geqslant 0\right)$, where $A d\left(u_{n}^{ \pm}\right)(a)=u_{n}^{ \pm} a\left(u_{n}^{ \pm}\right)^{*}$ for any $n \geqslant 0, a \in \mathcal{P}_{n}^{ \pm}$. By [7, Section 3], the map $u \in L \mapsto$ $\left(A d\left(u_{n}^{ \pm}\right): n \geqslant 0\right)$ is an embedding of the group $L$ into $\operatorname{Aut}(\mathcal{P})$.

Consider the group $\operatorname{Aut}(\Gamma, \mu)$ and its action $\gamma$ on $\mathcal{P}$. This action $\gamma$ defines an action of $\operatorname{Aut}(\Gamma, \mu)$ on $L$ that we continue to denote by $\gamma$. Consider the semi-direct product $L \rtimes \operatorname{Aut}(\Gamma, \mu)$ with respect to this action. Burstein proved that those two subgroups generates $\operatorname{Aut}(\mathcal{P})$ and that $\operatorname{Aut}(\mathcal{P})$ is isomorphic to $L \rtimes \operatorname{Aut}(\Gamma, \mu)$ [7]. We identify $\operatorname{Aut}(\mathcal{P})$ and $L \rtimes \operatorname{Aut}(\Gamma, \mu)$. If $g \in \operatorname{Aut}(\mathcal{P}), x \in \mathcal{P}_{n}^{ \pm}$, we denote by $g(x)$ the image of $x$ under the automorphism $g$.

We consider the action of $\operatorname{Aut}(\mathcal{P})$ on $V$ given by

$$
u g \cdot v=g v \text { for any } u \in U\left(\mathcal{P}_{1}^{+}\right), g \in \operatorname{Aut}(\Gamma, \mu) \text {, and } v \in V .
$$

Here are some remarks regarding fixed point spaces contained in $\mathcal{P}$.

Remark 3.1. Consider a $\operatorname{subgroup} G<\operatorname{Aut}(\mathcal{P})$ and the fixed point space $\mathcal{Q}=\mathcal{P}^{G}$ under the action of $G$.

- The collection of fixed point spaces $\left(\mathcal{Q}_{n}^{ \pm}=\left(\mathcal{P}_{n}^{ \pm}\right)^{G}: n \geqslant 0\right)$ is a planar algebra.

- If $G$ is contained in $\operatorname{Aut}(\Gamma, \mu)$ and acts transitively on $V^{+}$and $V^{-}$, then $\mathcal{Q}_{0}^{+}$and $\mathcal{Q}_{0}^{-}$are one dimensional and $\mathcal{Q}$ satisfies all the axioms of a subfactor planar algebra except the sphericality. In fact, $\mathcal{Q}$ is a subfactor planar algebra if and only if for any $a \in C_{1}^{ \pm}$we have that $\mu(a)\left|\left\{\alpha \in G \cdot a: s(\alpha)=v^{ \pm}\right\}\right|=\mu(\bar{a})\left|\left\{\alpha \in G \cdot a: t(\alpha)=v^{\mp}\right\}\right|$ where $v^{ \pm} \in V^{ \pm}$is a fixed pair of vertices. Indeed, the planar algebra $\mathcal{Q}$ is spherical if and only if $\tau_{r}$ and $\tau_{l}$ coincide on $\mathcal{Q}_{1}^{ \pm}$. Recall that $\left\{e_{a, b}:(a, b) \in S T_{1}^{ \pm}\right\}$is a system 
of matrix units of $\mathcal{P}_{1}^{ \pm}$, where $S T_{1}^{ \pm}=\left\{(a, b) \in C_{1}^{ \pm} \times C_{1}^{ \pm}: s(a)=s(b)\right.$ and $t(a)=$ $t(b)\}$. Therefore, $\mathcal{Q}_{1}^{ \pm}$is equal to the weak closure of $\operatorname{Span}\left\{f_{a, b}=\sum_{(\alpha, \beta) \in G \cdot(a, b)} e_{\alpha, \beta}\right.$ : $\left.(a, b) \in S T_{1}^{ \pm}\right\}$. Consider $(a, b) \in S T_{1}^{ \pm}$. We have that

$$
\begin{aligned}
\tau_{l}\left(f_{a, b}\right) & =\sum_{(\alpha, \beta) \in G \cdot(a, b)} \tau_{l}\left(e_{\alpha, \beta}\right)=\delta_{a, b} \sum_{\alpha \in G \cdot a} \mu(\bar{a}) e_{t(\alpha)}=\delta_{a, b} \sum_{w \in V^{\mp}} \sum_{\alpha \in G \cdot a: t(\alpha)=w} \mu(\bar{a}) e_{w} \\
& =\delta_{a, b} \mu(\bar{a})\left|\left\{\alpha \in G \cdot a: t(\alpha)=v^{\mp}\right\}\right| .
\end{aligned}
$$

A similar computation shows that $\tau_{r}\left(f_{a, b}\right)=\delta_{a, b} \mu(a)\left|\left\{\alpha \in G \cdot a: s(\alpha)=v^{ \pm}\right\}\right|$.

- If $\mathcal{P}^{G}$ is a subfactor planar algebra, then $G$ acts transitively on $V^{+}$and $V^{-}$.

- In general $\mathcal{Q}=\mathcal{P}^{G}$ is reducible. We can deduce the following. Suppose there exists a subgroup $G<\operatorname{Aut}(\mathcal{P})$ such that the fixed point space $\mathcal{P}^{G}$ is an irreducible subfactor planar algebra. Then, the group $\operatorname{Aut}(\Gamma, \mu)$ acts transitively on the set of positives edges $C_{1}^{+}$. This implies that the weight $\mu$ is constant on $C_{1}^{+}$.

- If we start with a countable locally finite undirected connected bipartite graph $\Gamma$ that can have multiple edges between two vertices and a group $G<\operatorname{Aut}(\Gamma)$ that acts transitively on $V^{+}$and on $V^{-}$, then it was observed in [2, Proposition 2.5] that there exists a unique weight $\mu: C^{1} \longrightarrow \mathbb{R}_{+}^{*}$ such that $\mathcal{P}^{G}$ is a subfactor planar algebra, where $\mathcal{P}$ is the bipartite graph planar algebra associated to $(\Gamma, \mu)$.

We fix a natural number $k \geqslant 0$.

Proposition 3.2. Let $j_{k}(n, m): \mathcal{P}_{n+m+2 k}^{+} \longrightarrow S_{k}$ be the inclusion of the vector space $\mathcal{P}_{n+m+2 k}^{+}$in $S_{k}$ for $n, m \geqslant 0$. There exists a group morphism $\sigma: \operatorname{Aut}(\mathcal{P}) \longrightarrow \operatorname{Aut}\left(S_{k}\right)$ that satisfies that $\sigma_{g} \circ j_{k}(n, m)(x)=j_{k}(n, m)(g(x))$ for any $g \in A u t(\mathcal{P}), n, m \geqslant 0$, and $x \in \mathcal{P}_{n+m+2 k}^{+}$. In particular, Aut $(\mathcal{P})$ acts on $T_{k}, M_{k}$ and $M_{k}^{o p}$. Moreover, the action of $\sigma$ is minimal, i.e. the relative commutant $\left(S_{k}^{\text {Aut }(\mathcal{P})}\right)^{\prime} \cap S_{k}$ is trivial.

Proof. For any $g \in \operatorname{Aut}(\mathcal{P}), n, m \geqslant 0, x \in \mathcal{P}_{n+m+2 k}^{+}$, we put $\sigma_{g}\left(j_{k}(n, m)(x)\right)=j_{k}(n, m)(g(x))$. Since any element $g \in \operatorname{Aut}(\mathcal{P})$ commutes with the action of the planar operad, we obtain that $\sigma_{g}$ is a $*$-algebra morphism of $G r_{k} \mathcal{P} \otimes G r_{k} \mathcal{P}$. Observe that $p_{v} H_{k}$ is orthogonal to $p_{w} H_{k}$ for two different vertices $v, w \in V^{+}$. Since $\sum_{v \in V^{+}} p_{v}=1$, we obtain that

$$
H_{k}=\bigoplus_{v \in V^{+}} p_{v} H_{k}
$$

Consider $g \in \operatorname{Aut}(\Gamma, \mu)$ and $x \in G r_{k} \mathcal{P} \otimes G r_{k} \mathcal{P}$ such that $\left\{v \in V: p_{v} x \neq 0\right\}$ is finite. Note that $\operatorname{Tr}\left(x x^{*}\right)<\infty$. Observe that $\operatorname{Tr} \circ \sigma_{g}=c_{g} \cdot \operatorname{Tr}$ for any $g \in G$, where $c_{g}$ is the ratio $\frac{\operatorname{Tr}\left(p_{g w}\right)}{\operatorname{Tr}\left(p_{w}\right)}=\frac{\mu_{V}(g w)^{2}}{\mu_{V}(w)^{2}}$ which does not depend on $w \in V^{+}$. We put $U_{g}(x)=\sigma_{g}(x) / \sqrt{c_{g}}$. Observe that

$$
c_{g}\left\|U_{g}(x)\right\|_{2}^{2}=\left\|\sigma_{g}(x)\right\|_{2}^{2}=\tau_{V} \circ E \circ \sigma_{g}\left(x x^{*}\right)=\tau_{V} \circ \sigma_{g} \circ E\left(x x^{*}\right),
$$

since $g$ commutes with the action of the planar operad. Since $\tau_{V} \circ \sigma_{g}=c_{g} \cdot \tau_{V}$, we obtain that $\left\|U_{g}(x)\right\|_{2}^{2}=\tau_{V} \circ E\left(x x^{*}\right)=\|x\|_{2}^{2}$. This implies that $U_{g}$ extends as an isometry of $H_{k}$. By definition, $U_{g^{-1}} \circ U_{g}(x)=x$ for any $n, m \geqslant 0$ and $x$ in the range of $j_{k}(n, m)$. This implies that $U_{g^{-1}} \circ U_{g}$ is the identity operator. By symmetry, $U_{g}$ is a unitary of $H_{k}$ and $U_{g}^{*}=U_{g^{-1}}$. By definition and by a density argument we obtain that $U_{g} U_{h}=U_{g h}$ for any $g, h \in \operatorname{Aut}(\Gamma, \mu)$. Consider the map $A d\left(U_{g}\right)(x)=U_{g} x U_{g}^{*}$ for any $g \in \operatorname{Aut}(\Gamma, \mu), x \in B\left(H_{k}\right)$. Consider $x \in G r_{k} \mathcal{P} \otimes G r_{k} \mathcal{P}, g \in \operatorname{Aut}(\Gamma, \mu)$, and $\xi \in H_{k} \cap G r_{k} \mathcal{P} \otimes G r_{k} \mathcal{P}$. We have that

$$
A d\left(U_{g}\right)(x) \xi=U_{g} x U_{g}^{*} \xi=U_{g} x \sqrt{c_{g}} \sigma_{g^{-1}}(\xi)=\sigma_{g}\left(x \sigma_{g^{-1}}(\xi)\right)=\sigma_{g}(x) \xi .
$$


If $g \in L$, we define $U_{g}(x)=\sigma_{g}(x)$ for any $x \in H_{k} \cap G r_{k} \mathcal{P} \otimes G r_{k} \mathcal{P}$. A similar proof shows that $\operatorname{Ad}\left(U_{g}\right)(x)=\sigma_{g}(x)$ for any $x \in G r_{k} \mathcal{P} \otimes G r_{k} \mathcal{P}, g \in L$. One can check that $U_{g} U_{l} U_{g}^{*}=U_{\gamma_{g}(l)}$ for any $g \in \operatorname{Aut}(\Gamma, \mu), l \in L$. This implies that $U: \operatorname{Aut}(\mathcal{P}) \longrightarrow U\left(H_{k}\right)$ is a unitary representation. By density, we obtain that $\operatorname{Ad}\left(U_{g}\right)\left(S_{k}\right)=S_{k}$ for any $g \in \operatorname{Aut}(\mathcal{P})$. We denote by $\sigma_{g}$ the restriction to $S_{k}$ of the automorphism $\operatorname{Ad}\left(U_{g}\right), g \in \operatorname{Aut}(\mathcal{P})$. We have that $\sigma_{g} \circ j_{k}(n, m)(x)=j_{k}(n, m)(g(x))$ for any $g \in \operatorname{Aut}(\mathcal{P}), n, m \geqslant 0$, and $x \in \mathcal{P}_{n+m+2 k}^{+}$. Hence, $\operatorname{Aut}(\mathcal{P})$ acts on $T_{k}, M_{k}$ and $M_{k}^{\text {op }}$ by restricting $\sigma_{g}, g \in \operatorname{Aut}(\mathcal{P})$.

If $\mathcal{J}$ is the Temperley-Lieb-Jones planar algebra with modulus $\delta$, then $S_{k}(\mathcal{J})$ is contained in the fixed point space $S_{k}^{\operatorname{Aut}(\mathcal{P})}$. By Proposition $2.3, S_{k}(\mathcal{J})$ is an irreducible subfactor of $S_{k}$. Therefore, $S_{k}^{\operatorname{Aut}(\mathcal{P})} \subset S_{k}$ is an irreducible subfactor.

Proposition 3.3. Consider a subgroup $G<A u t(\mathcal{P})$ and the fixed point planar algebra $\mathcal{Q}:=\mathcal{P}^{G}$. Assume that $\mathcal{Q}$ is a subfactor planar algebra. Consider the fixed point von Neumann algebras $S_{k}^{G}, M_{k}^{G}$, and $\left(M_{k}^{o p}\right)^{G}$. We have the equalities $S_{k}^{G}=S_{k}(\mathcal{Q}), M_{k}^{G}=M_{k}(\mathcal{Q})$, and $\left(M_{k}^{o p}\right)^{G}=M_{k}^{o p}(\mathcal{Q})$, where $S_{k}(\mathcal{Q}), M_{k}(\mathcal{Q})$, and $M_{k}^{o p}(\mathcal{Q})$ are the von Neumann subalgebras of $S_{k}$ defined in Section [2.3. In particular, $M_{0}^{G} \subset M_{1}^{G}$ is a subfactor with subfactor planar algebra isomorphic to $\mathcal{Q}$. Furthermore, the symmetric enveloping inclusion of $\mathcal{Q}$ is isomorphic to $M_{0}^{G} \vee\left(M_{0}^{o p}\right)^{G} \subset S_{0}^{G}$.

Proof. We assume that $k=0$ and drop the subscript $k$. The general case can easily be deduced. Let us show that $S^{G}=S(\mathcal{Q})$. By definition, $S(\mathcal{Q})$ is the weak closure of $B:=\operatorname{Gr} \mathcal{Q} \otimes G r \mathcal{Q}$ inside $S$. Any element of $B$ is $G$-invariant. Therefore, $B$ is included in $S^{G}$ and so does its weak closure $S(\mathcal{Q})$.

Consider a vertex $o \in V^{+}$and the normal state $\operatorname{tr}: S \longrightarrow \mathbb{C}, x \longmapsto \operatorname{Tr}\left(p_{o}\right)^{-1} \operatorname{Tr}\left(x p_{o}\right)$. By Proposition 2.4, $S(\mathcal{Q})$ is a $\mathrm{II}_{1}$ factor and $t r$ is its unique normal faithful tracial state. In particular, $t r$ is faithful on $S(\mathcal{Q})$. Let us show that $t r$ is faithful on $S^{G}$. Consider $x \in S^{G}$ such that $\operatorname{tr}\left(x x^{*}\right)=0$. The action of $G$ on $V^{+}$is transitive since $\mathcal{P}^{G}$ is a subfactor planar algebra. Hence, for any $v \in V^{+}$, there exists $g_{v} \in G$ such that $g o=v$. Observe that

$$
\begin{aligned}
\operatorname{Tr}\left(x x^{*}\right) & =\sum_{v \in V^{+}} \operatorname{Tr}\left(x x^{*} p_{v}\right)=\sum_{v \in V^{+}} \operatorname{Tr}\left(x x^{*} p_{g_{v} o}\right) \\
& =\sum_{v \in V^{+}} \operatorname{Tr}\left(\sigma_{g_{v}}\left(x x^{*} p_{o}\right)\right) \text { since } x x^{*} \text { is } G \text {-invariant } \\
& =\sum_{v \in V^{+}} \frac{\mu_{V}(v)^{2}}{\mu_{V}(o)^{2}} \operatorname{Tr}\left(x x^{*} p_{o}\right)=\sum_{v \in V^{+}} \mu_{V}(v)^{2} \operatorname{tr}\left(x x^{*}\right)=0 .
\end{aligned}
$$

Since $\operatorname{Tr}$ is faithful, we obtain that $x=0$. Therefore, $\operatorname{tr}$ is a normal faithful state on $S^{G}$.

Let us show that $L^{2}(S(\mathcal{Q}), t r)=L^{2}\left(S^{G}, t r\right)$, where $L^{2}(S(\mathcal{Q}), t r)$ and $L^{2}\left(S^{G}, t r\right)$ are the GNS Hilbert space associated to $(S(\mathcal{Q}), t r)$ and $\left(S^{G}, t r\right)$. Consider $\xi$ in the orthogonal complement of $L^{2}(S(\mathcal{Q}), t r)$ inside $L^{2}\left(S^{G}, t r\right)$. Observe that $\operatorname{Tr}\left(\left(p_{o} \xi\right)\left(p_{o} \xi\right)^{*}\right)=\operatorname{Tr}\left(\xi \xi^{*} p_{o}\right)=$ $\operatorname{Tr}\left(p_{o}\right) \operatorname{tr}\left(\xi \xi^{*}\right)<\infty$. Therefore, $p_{o} \xi \in L^{2}(S, \operatorname{Tr})$. The Hilbert space $L^{2}(S, \operatorname{Tr})$ is equal to the direct sum

$$
\bigoplus_{n, m \geqslant 0} H(n, m)
$$

Hence, there exists $x_{m}^{n} \in H(n, m), n, m \geqslant 0$ such that $p_{0} \xi=\sum_{n, m \geqslant 0} x_{m}^{n}$ where the sum converges in $L^{2}(S, T r)$. Let us fixed $n, m \geqslant 0$. We have that $x_{m}^{n} \in p_{o} H(n, m)$. Observe 
that

$$
\left\{j(n, m)\left(e_{a, b}\right):(a, b) \in S T_{n+m}^{+}, s(a)=o\right\}
$$

is an orthogonal basis of $p_{o} H(n, m)$. Since $\Gamma$ is locally finite, we obtain that this basis is finite. Therefore, $p_{o} H(n, m)$ is finite dimensional and is equal to $p_{o} D(n, m)$. Hence, there exists a unique $d \in e_{o} \mathcal{P}_{n+m}^{+}$such that $j(n, m)(d)=x_{m}^{n}$. Recall that

$\mathcal{P}_{n+m}^{+}=\bigoplus_{v \in V^{+}, w \in V} B\left(\ell^{2}\left(C_{n+m}^{+}(v, w)\right)\right)$, where $C_{n+m}^{+}(v, w)=\left\{a \in C_{n+m}^{+}: s(a)=v, t(a)=w\right\}$.

We have that $d \in \bigoplus_{w \in V} B\left(\ell^{2}\left(C_{n+m}^{+}(o, w)\right)\right)$.

If $g \in \operatorname{Aut}(\mathcal{P})$, then $g(d) \in \bigoplus_{w \in V} B\left(\ell^{2}\left(C_{n+m}^{+}(g o, w)\right)\right)$. Consider $G_{o}=\{g \in G: g o=$ $o\}$, and a system of representatives $\left\langle G / G_{o}\right\rangle$ of the quotient space $G / G_{o}$. The elements $p_{o}$ and $\xi$ are $G_{o}$-invariants. Therefore, $p_{o} \xi$ is $G_{o}$-invariant as an element of $L^{2}(S, T r)$. Consider the orthogonal projection $P: L^{2}(S, T r) \longrightarrow H(n, m)$ and observe that $P$ is $G$-equivariant. Consider $g \in G_{o}$. Since $g$ fixes $o$, we have that $\operatorname{Tr} \circ \sigma_{g}=\operatorname{Tr}$ by uniqueness of the trace. Hence, $\sigma_{g}$ and $U_{g}$ coincide on $L^{2}(S, T r) \cap S$, where $U_{g}$ is the unitary defined in the proof of the previous proposition. Observe that $j(n, m)(g(d))=\sigma_{g} \circ j(n, m)(d)=$ $U_{g}\left(P\left(p_{o} \xi\right)\right)=P \circ U_{g}\left(p_{o} \xi\right)=P\left(p_{o} \xi\right)=j(n, m)(d)$. Therefore, $d$ is $G_{o}$-invariant as an element of $\mathcal{P}_{n+m}^{+}$.

We have that $g o \neq h o$ for any $g \neq h$ in $\left\langle G / G_{o}\right\rangle$. This implies that the elements of $\left\{g(d): g \in\left\langle G / G_{o}\right\rangle\right\}$ belongs to distinct summands of the von Neumann algebra

$$
\mathcal{P}_{n+m}^{+}=\bigoplus_{v \in V^{+}, w \in V} B\left(\ell^{2}\left(C_{n+m}^{+}(v, w)\right)\right) .
$$

Moreover, $\|g(d)\|=\|d\|$ for any $g \in \operatorname{Aut}(\mathcal{P})$ since $g$ is an automorphism of the von Neumann algebra $\mathcal{P}_{n+m}^{+}$. This implies that the sum $\sum_{g \in\left\langle G / G_{o}\right\rangle} g(d)$ converges for the strong operator topology to an element $\tilde{d} \in \mathcal{P}_{n+m}^{+}$. Let us show that $\tilde{d}$ is $G$-invariant. Consider $f=\sum_{r \in R} r(d)$, where $R$ is a system of representatives of $G / G_{o}$. For any $r \in R$ there exists $g_{r} \in\left\langle G / G_{o}\right\rangle, h_{r} \in G_{o}$ such that $r=g_{r} h_{r}$. Moreover, $\left\{g_{r}: r \in R\right\}=\left\langle G / G_{o}\right\rangle$. Observe that

$$
f=\sum_{r \in R} r(d)=\sum_{r \in R} g_{r} h_{r}(d)=\sum_{r \in R} g_{r}(d)=\sum_{g \in\left\langle G / G_{o}\right\rangle} g(d)=\tilde{d} .
$$

Therefore, $\tilde{d}$ does not depend on the choice of the system of representatives $\left\langle G / G_{o}\right\rangle$. Consider $g \in G$. We have that $g(\tilde{d})=\sum_{h \in\left\langle G / G_{o}\right\rangle} g h(d)=\sum_{r \in R} r(d)$, where $R=\{g h: h \in$ $\left.\left\langle G / G_{o}\right\rangle\right\}$. The set $R$ is a system of representatives of $G / G_{o}$. Therefore, $g(\tilde{d})=\tilde{d}$. Hence, $\tilde{d}$ is a $G$-invariant element of $\mathcal{P}_{n+m}^{+}$. This means that $\tilde{d} \in \mathcal{Q}_{n+m}^{+}$.

We put $y_{m}^{n}=j(n, m)(\tilde{d})$. By definition, $y_{m}^{n} \in B$. By assumption, $\operatorname{tr}\left(\xi\left(y_{m}^{n}\right)^{*}\right)=0$. Observe that

$$
\begin{aligned}
\operatorname{Tr}\left(p_{o}\right) \operatorname{tr}\left(\xi \xi^{*}\right) & =\operatorname{Tr}\left(\xi \xi^{*} p_{o}\right)=\operatorname{Tr}\left(\xi\left(p_{o} \xi\right)^{*}\right)=\sum_{k, l \geqslant 0} \operatorname{Tr}\left(\xi\left(x_{l}^{k}\right)^{*}\right) \\
& =\sum_{k, l \geqslant 0} \operatorname{Tr}\left(\xi\left(y_{l}^{k}\right)^{*} p_{o}\right) \text { since } p_{o} y_{l}^{k}=x_{l}^{k} \\
& =\operatorname{Tr}\left(p_{o}\right) \sum_{k, l \geqslant 0} \operatorname{tr}\left(\xi\left(y_{l}^{k}\right)^{*}\right)=0 .
\end{aligned}
$$


We obtain that $L^{2}(S(\mathcal{Q}), t r)=L^{2}\left(S^{G}, t r\right)$ since the orthogonal complement of $L^{2}(S(\mathcal{Q}), t r)$ inside $L^{2}\left(S^{G}, t r\right)$ is trivial. Consider $\xi \in S^{G}$. Since $t r$ is a faithful state, we have that $\xi \in L^{2}\left(S^{G}, t r\right)$. Hence, $\xi \in L^{2}(S(\mathcal{Q}), t r)$. Let us show that $\xi$ is a bounded vector of the $\mathrm{II}_{1}$ factor $S(\mathcal{Q})$. Consider the map $\theta: S(\mathcal{Q}) \longrightarrow L^{2}(S(\mathcal{Q}), t r), y \longmapsto y \xi$. Observe that

$$
\begin{aligned}
\operatorname{tr}\left(\theta(y) \theta(y)^{*}\right) & =\operatorname{Tr}\left(p_{o}\right)^{-1} \operatorname{Tr}\left(y \xi \xi^{*} y^{*} p_{o}\right) \leqslant \operatorname{Tr}\left(p_{o}\right)^{-1}\left\|\xi \xi^{*}\right\|_{S} \operatorname{Tr}\left(y y^{*} p_{o}\right) \\
& \leqslant\|\xi\|_{S}^{2} \operatorname{tr}\left(y y^{*}\right) \text { for any } y \in S(\mathcal{Q}) .
\end{aligned}
$$

Therefore, $\theta$ extends as a bounded linear operator from $L^{2}(S(\mathcal{Q}), t r)$ to $L^{2}(S(\mathcal{Q}), \operatorname{tr})$. Since $S(\mathcal{Q})$ is a $\mathrm{II}_{1}$ factor and $t r$ is its unique normal faithful tracial state, we obtain that $\xi \in S(\mathcal{Q})$ by [18, Theorem 1.2.4.2]. Therefore, $S(\mathcal{Q})=S^{G}$.

A similar proof show that $M(\mathcal{Q})=M^{G}$ and $M(\mathcal{Q})^{\mathrm{op}}=\left(M^{\mathrm{op}}\right)^{G}$. By [11] and Proposition 2.5, $\mathcal{Q}$ is isomorphic to the subfactor planar algebra of $M_{0}(\mathcal{Q}) \subset M_{1}(\mathcal{Q})$. Therefore, it is isomorphic to the subfactor planar algebra of $M_{0}^{G} \subset M_{1}^{G}$. The symmetric enveloping inclusion of $\mathcal{Q}$ is isomorphic to $M_{0}(\mathcal{Q}) \vee M_{0}(\mathcal{Q})^{\mathrm{op}} \subset S_{0}(\mathcal{Q})$ by Proposition 2.5. Therefore, it is isomorphic to $M_{0}^{G} \vee\left(M_{0}^{\mathrm{op}}\right)^{G} \subset S_{0}^{G}$.

3.2. Proof of Theorem $\mathbf{A}$. Let $(\Gamma, \mu)$ be a weighted graph with modulus $\delta$ and let $G<\operatorname{Aut}(\mathcal{P})$ be a countable or closed subgroup. Let $\mathcal{P}$ be the bipartite graph planar algebra associated to $(\Gamma, \mu)$ and let $T \subset S$ be its symmetric enveloping inclusion. Consider the fixed point space $\mathcal{Q}=\mathcal{P}^{G}$ that we assume to be a subfactor planar algebra. This implies that the action of $G$ on $V^{+}$is necessarily transitive and that $T \subset S$ is an irreducible subfactor. If $K<L$ is an inclusion of groups, we denote by $\langle L / K\rangle$ (resp. $\langle K \backslash L / K\rangle)$ a system of representatives of the coset space $L / K$ (resp. $K \backslash L / K)$. If $v, w \in V^{+}$, then $G_{v}$ (resp. $G_{v, w}$ ) denotes the subgroup of $G$ that fixes the vertex $v$ (resp. fixes the vertices $v$ and $w)$. Let $o \in V^{+}$be a fixed vertex. Note that $G / G_{o}$ is in bijection with $V^{+}$since $G$ acts transitively on $V^{+}$. Hence, $\left\{p_{g o}: g \in\left\langle G / G_{o}\right\rangle\right\}$ is the set of minimal central projections of $T$.

Remark 3.4. - The subgroup $G_{o}<G$ is almost normal. Indeed, consider $g \in G$. We need to show that $G_{o} \cap g G_{o} g^{-1}$ is a finite index subgroup of $G_{o}$ and $g G_{o} g^{-1}$. Observe that $G_{o} \cap g G_{o} g^{-1}=G_{o, g o}=\{h \in G: h g o=g o$ and $h o=o\}$. Consider the map $h \in G_{o} \longmapsto h g o \in V$. We have that $G_{o} / G_{o, g o}$ is in bijection with the orbit $G_{o} \cdot g o$. But $G_{o} \cdot g o$ is contained in the sphere centered in $o$ with radius equal to the distance between $o$ and $g o$. This sphere is a finite set since $\Gamma$ is locally finite. Therefore, $G_{o, g o}$ is a finite index subgroup of $G_{o}$. A similar argument shows that $G_{o, g o}$ is a finite index subgroup of $g G_{o} g^{-1}$. Therefore, $G_{o}<G$ is an almost normal subgroup.

- By Propositions 2.4 and 3.3 , the unique normal faithful tracial state of the $\mathrm{II}_{1}$ factor $S^{G}$ is $\operatorname{tr}: S^{G} \longrightarrow \mathbb{C}, x \mapsto \operatorname{Tr}\left(p_{v}\right)^{-1} \operatorname{Tr}\left(p_{v} x\right)$ where $v \in V^{+}$.

The next proposition gives a decomposition of the $T^{G}$-bimodule $L^{2}\left(S^{G}, t r\right)$.

Proposition 3.5. We define the Hilbert space $H_{g}$ which is the closure in $L^{2}(S, T r)$ of $p_{o} S^{G} p_{\text {go }}$ for any $g \in G$. The following assertions are true.

(1) The Hilbert space $H_{g}$ is a bifinite $T^{G}$-bimodule for any $g \in G$.

(2) The $T^{G}$-bimodules $L^{2}\left(S^{G}, t r\right)$ and $\bigoplus_{g \in\left\langle G_{o} \backslash G / G_{o}\right\rangle} H_{g}$ are isomorphic.

Proof. Proof of (1). Consider $g \in G$. Since $\operatorname{Tr}\left(p_{o}\right)<\infty$, we have that $p_{o} S$ is contained in $L^{2}(S, T r)$. Therefore, $H_{g}$ is well defined. Since $p_{g o}$ and $p_{o}$ commute with $T^{G}$, 
we have that $H_{g}$ is a $T^{G}$-bimodule. Suppose that $\operatorname{Tr}\left(p_{o}\right) \leqslant \operatorname{Tr}\left(p_{g o}\right)$. Since $S^{G_{o, g o}}$ is a factor by Proposition 3.2, there exists some elements $m_{1}, \cdots, m_{n} \in S^{G_{o, g o}}$ such that $m_{1} m_{1}^{*}=p_{o}, m_{i} m_{i}^{*} \leqslant p_{o}, 2 \leqslant i \leqslant n$ and $\sum_{j=1}^{n} m_{j}^{*} m_{j}=p_{g o}$. Since $G_{o}<G$ is an almost normal subgroup by Remark 3.4, we have that the index $\left[G_{o}: G_{o, g_{o}}\right]$ is finite. Therefore, $\left[T(o)^{G_{o, g o}}: T(o)^{G_{o}}\right]<\infty$. Hence, there exists a finite subset $F \subset T(o)^{G_{o, g o}}$ such that $T(o)^{G_{o, g o}}=\operatorname{Span}\left(T(o)^{G_{o}} \cdot F\right)$. Similarly, there exists a finite subset $E \subset T(g o)^{G_{o, g o}}$ such that $T\left(g_{o}\right)^{G_{o, g o}}=\operatorname{Span}\left(E \cdot T(g o)^{G_{g o}}\right)$. Observe that

$$
p_{g o} T^{G}=T^{G} p_{g o}=T(g o)^{G_{g o}} .
$$

Consider $x \in p_{o} S^{G} p_{g o}$. We have that $x=m_{1}\left(m_{1}^{*} x\right)$ and $m_{1}^{*} x \in p_{g o} S p_{g o}=T(g o)$. Moreover, $m_{1}^{*} x$ is $G_{o, g o}$-invariant. Therefore, $m_{1}^{*} x$ is in $T(g o)^{G_{o, g o}}=\operatorname{Span}\left(E \cdot T(g o)^{G_{g o}}\right)$. Hence, $x \in \operatorname{Span}\left(m_{1} \cdot E \cdot T(g o)^{G_{g o}}\right)=\operatorname{Span}\left(m_{1} \cdot E \cdot T^{G}\right)$.

Consider $1 \leqslant i \leqslant n$. Observe that $x=\sum_{j=1}^{n} x m_{j}^{*} m_{j}$ and $x m_{i}^{*} \in p_{o} S p_{o}=T(o)$. Moreover, $x m_{i}^{*}$ is $G_{o, g o}$-invariant. Hence, $x m_{i}^{*}$ is in $T(o)^{G_{o, g o}}=\operatorname{Span}\left(T(o)^{G_{o}} \cdot F\right)=$ $\operatorname{Span}\left(T^{G} \cdot F\right)$. Therefore, $x$ is in $\operatorname{Span}\left(T^{G} \cdot F \cdot\left\{m_{j}: 1 \leqslant j \leqslant n\right\}\right)$. Therefore, $H_{g}$ is a bifinite $T^{G}$-bimodule.

Proof of (2). Consider the map $\beta: L^{2}\left(S^{G}, t r\right) \longrightarrow L^{2}(S, T r)$ such that

$$
\beta(x)=\sum_{g \in\left\langle G_{o} \backslash G / G_{o}\right\rangle} p_{o} x p_{g o} \sqrt{\frac{\left[G_{o}: G_{o, g o}\right]}{\operatorname{Tr}\left(p_{o}\right)}}
$$

for any $x \in S^{G}$, where the sum converges for the $L^{2}$-norm. Let us show that $\beta$ is an isometry. Let $\sigma: G \longrightarrow \operatorname{Aut}(S)$ be the action of $G$ on $S$. Observe that $\operatorname{Tr} \circ \sigma_{g}=c_{g} \cdot \operatorname{Tr}$ for any $g \in G$, where $c_{g}$ is the ratio $\frac{\operatorname{Tr}\left(p_{g w}\right)}{\operatorname{Tr}\left(p_{w}\right)}$ which does not depend on $w \in V^{+}$. In particular,

$$
\operatorname{Tr} \circ \sigma_{g}=\operatorname{Tr} \text { if } g \in G \text { fixes a vertex of } V^{+} \text {. }
$$

Consider $x \in S^{G}$. We have that

$$
\begin{aligned}
\operatorname{Tr}\left(\beta(x) \beta(x)^{*}\right) & =\sum_{g, h \in\left\langle G_{o} \backslash G / G_{o}\right\rangle} \frac{\sqrt{\left[G_{o}: G_{o, g o}\right]\left[G_{o}: G_{o, h o}\right]}}{\operatorname{Tr}\left(p_{o}\right)} \operatorname{Tr}\left(p_{o} x p_{g o} p_{h o} x^{*} p_{o}\right) \\
& =\sum_{g \in\left\langle G_{o} \backslash G / G_{o}\right\rangle} \frac{\left[G_{o}: G_{o, g o}\right]}{\operatorname{Tr}\left(p_{o}\right)} \operatorname{Tr}\left(p_{o} x p_{g o} x^{*}\right) .
\end{aligned}
$$

Consider $g, h \in G$ such that $G_{o} g G_{o}=G_{o} h G_{o}$. Then, there exists $k \in G_{o}$ such that $g G_{o}=k h G_{o}$. Observe that

$$
\begin{aligned}
\operatorname{Tr}\left(p_{o} x p_{g o} x^{*}\right) & =\operatorname{Tr}\left(p_{o} x p_{k h o} x^{*}\right)=\operatorname{Tr} \circ \sigma_{k}\left(p_{o} x p_{h o} x^{*}\right) \\
& \left.=\operatorname{Tr}\left(p_{o} x p_{h o} x^{*}\right) \text { by (10) }\right) .
\end{aligned}
$$


Therefore, $\operatorname{Tr}\left(p_{o} x p_{g o} x^{*}\right)=\left[G_{o}: G_{o, g o}\right]^{-1} \sum_{k \in\left\langle G_{o} / G_{o, g o}\right\rangle} \operatorname{Tr}\left(p_{o} x p_{k g o} x^{*}\right)$. Hence,

$$
\begin{aligned}
\operatorname{Tr}\left(\beta(x) \beta(x)^{*}\right) & =\sum_{g \in\left\langle G_{o} \backslash G / G_{o}\right\rangle} \sum_{k \in\left\langle G_{o} / G_{o, g o}\right\rangle} \frac{\operatorname{Tr}\left(p_{o} x p_{k g o} x^{*}\right)}{\operatorname{Tr}\left(p_{o}\right)} \\
& =\sum_{s \in\left\langle G / G_{o}\right\rangle} \frac{\operatorname{Tr}\left(p_{o} x p_{s o} x^{*}\right)}{\operatorname{Tr}\left(p_{o}\right)} \text { for a system of representatives }\left\langle G / G_{o}\right\rangle \\
& =\frac{\operatorname{Tr}\left(p_{o} x x^{*}\right)}{\operatorname{Tr}\left(p_{o}\right)}=\operatorname{tr}\left(x x^{*}\right) .
\end{aligned}
$$

Therefore, $\beta$ defines an isometry from $L^{2}\left(S^{G}, t r\right)$ to $L^{2}(S, T r)$.

The map $\beta$ is $T^{G}$-bimodular because $p_{o}$ and $p_{g o}$ commute with $T^{G}$ for any $g \in G$. Note that $H_{g}$ is orthogonal to $H_{k}$ if $g G_{o} \neq k G_{o}$. Therefore, the $T^{G}$-bimodules $\left\{H_{l}: l \in\right.$ $\left.\left\langle G_{o} \backslash G / G_{o}\right\rangle\right\}$ are pairwise orthogonal. We have that $\operatorname{Im} \beta \subset \bigoplus_{l \in\left\langle G_{o} \backslash G / G_{o}\right\rangle} H_{l}$ by definition. Let us show that the range of $\beta$ is equal to $\bigoplus_{l \in\left\langle G_{o} \backslash G / G_{o}\right\rangle} H_{l}$.

We fix $g \in\left\langle G_{o} \backslash G / G_{o}\right\rangle$. Consider $y \in p_{o} S^{G} p_{g o}=H_{g} \cap S$. Let us show that the sum $\sum_{s \in\left\langle G / G_{o, g o}\right\rangle} \sigma_{s}(y)$ converges for the weak operator topology of $S$. Consider $\varepsilon>0, \xi, \eta \in$ $L^{2}(S, T r)$ such that $\|\xi\|_{2}=\|\eta\|_{2}=1$. If $A \subset V^{+}$, we denote by $A^{c}$ its complement and by $p_{A}$ the projection $\sum_{v \in A} p_{v}$. Since $1=\|\xi\|_{2}^{2}=\sum_{v \in V^{+}}\left\|p_{v} \xi\right\|_{2}^{2}=\sum_{v \in V^{+}}\left\|p_{v} \eta\right\|_{2}^{2}$, there exists a finite subset $A \subset V^{+}$such that $\left\|p_{A^{c}} \xi\right\|_{2}^{2},\left\|p_{A^{c}} \eta\right\|_{2}^{2}<\varepsilon$. Consider the set $E=\left\{s \in\left\langle G / G_{o, g o}\right\rangle: s o \in A\right.$ or sgo $\left.\in A\right\}$. Note that its cardinal $|E|$ is smaller than $|A|\left(\left[G_{o}: G_{o, g o}\right]+\left[G_{g o}: G_{o, g o}\right]\right)<\infty$. Denote by $E^{c}$ the complement of $E$ inside $\left\langle G / G_{o, g o}\right\rangle$. Observe that

$$
\begin{aligned}
\left|\left\langle\sum_{s \in E^{c}} \sigma_{s}(y) \xi, \eta\right\rangle\right| & \leqslant \sum_{s \in E^{c}}\left|\left\langle\sigma_{s}(y) \xi, \eta\right\rangle\right| \leqslant \sum_{s \in E^{c}}\left|\left\langle\sigma_{s}(y) p_{s g o} \xi, p_{s o} \eta\right\rangle\right| \text { since } y \in p_{o} S p_{g o} \\
& \leqslant \sum_{s \in E^{c}}\left\|\sigma_{s}(y) p_{s g o} \xi\right\|_{2}\left\|p_{s o} \eta\right\|_{2} \text { by the Cauchy-Schwarz inequality } \\
& \leqslant \sum_{s \in E^{c}}\|y\|\left\|p_{s g o} \xi\right\|_{2}\left\|p_{s o} \eta\right\|_{2} \\
& \leqslant\|y\| \sqrt{\sum_{s \in E^{c}}\left\|p_{s g o} \xi\right\|_{2}^{2}} \sqrt{\sum_{t \in E^{c}}\left\|p_{t o} \eta\right\|_{2}^{2}} \text { by the Cauchy-Schwarz inequality } \\
& \leqslant\|y\| \sqrt{\sum_{w \in A^{c}}\left|\left\{s \in E^{c}: s g o=w\right\}\right|\left\|p_{w} \xi\right\|_{2}^{2}} \sqrt{\sum_{w \in A^{c}}\left|\left\{t \in E^{c}: t o=w\right\}\right|\left\|p_{w} \eta\right\|_{2}^{2}}
\end{aligned}
$$

Consider $w \in V^{+}, r \in G$ such that $r w=o$ and $F=\left\{t \in E^{c}: t o=w\right\}$. Observe that $r \cdot F \subset G_{o} \cap r\left\langle G / G_{o, g_{o}}\right\rangle$. Therefore, $|F|=|r \cdot F| \leqslant\left|G_{o} \cap r\left\langle G / G_{o, g o}\right\rangle\right|=\left[G_{o}: G_{o, g o}\right]$, since $\left|G_{o} \cap R\right|=\left[G_{o}: G_{o, g_{o}}\right]$ for any system of representatives $R$ of $G / G_{o, g_{o}}$. Similarly, $\left|\left\{s \in E^{c}: s g o=w\right\}\right| \leqslant\left[G_{g o}: G_{o, g o}\right]$. Therefore,

$$
\begin{aligned}
\left|\left\langle\sum_{s \in E^{c}} \sigma_{s}(y) \xi, \eta\right\rangle\right| & \leqslant \sqrt{\left[G_{o}: G_{o, g o}\right]\left[G_{g o}: G_{o, g o}\right]}\left\|p_{A^{c}} \xi\right\|_{2}\left\|p_{A^{c}} \eta\right\|_{2} \\
& \leqslant \varepsilon \sqrt{\left[G_{o}: G_{o, g o}\right]\left[G_{g o}: G_{o, g o}\right]} .
\end{aligned}
$$


This implies that for any $\delta>0$ there exists a finite set $E \subset\left\langle G / G_{o, g_{o}}\right\rangle$ such that for any sets $I, J$ that contains $E$ we have that $\left|\left\langle\sum_{s \in I} \sigma_{s}(y) \xi, \eta\right\rangle-\left\langle\sum_{t \in J} \sigma_{t}(y) \xi, \eta\right\rangle\right|<\delta$. Hence, $\left(\left(\sum_{s \in I} \sigma_{s}(y): I \subset\left\langle G / G_{o, g o}\right\rangle\right)\right.$ is a Cauchy filter in $S$ with respect to the weak uniform structure. This implies that the sum $\sum_{s \in\left\langle G / G_{o, g o}\right\rangle} \sigma_{s}(y)$ converges weakly in $S$, since $S$ is complete for the weak uniform structure. We denote by $\Theta_{g}(y)$ this limit. Let us show that $\Theta_{g}(y)$ is $G$-invariant. Suppose that $R$ is a system of representatives of $G / G_{o, g o}$. For any $r \in R$ there exists a unique $a_{r} \in\left\langle G / G_{o, g o}\right\rangle$ and $b_{r} \in G_{o, g o}$ such that $r=a_{r} b_{r}$. we have that $\sigma_{r}(y)=\sigma_{a_{r} b_{r}}(y)=\sigma_{a_{r}} \circ \sigma_{b_{r}}(y)=\sigma_{a_{r}}(y)$ since $y$ is $G_{o, g o}$-invariant. Moreover, $\left\{a_{r}: r \in R\right\}=\left\langle G / G_{o, g o}\right\rangle$. Therefore, $\Theta_{g}(y)=\sum_{r \in R} \sigma_{r}(y)$. Hence, $\Theta_{g}$ does not depend on the choice of the system of representatives of $G / G_{o, g o}$. Consider $a \in G$. We have that $\sigma_{a} \circ \Theta_{g}(y)=\sum_{r \in R} \sigma_{r}(y)$, where $R=a \cdot\left\langle G / G_{o, g o}\right\rangle$. Since $R$ is a system of representatives, we obtain that $\sigma_{a} \circ \Theta_{g}(y)=\Theta_{g}(y)$. Hence, $\Theta_{g}(y)$ is $G$-invariant.

Let $\left\langle G_{o} / G_{o, g o}\right\rangle$ be a system of representatives of $G_{o} / G_{o, g o}$. Observe that

$$
\begin{aligned}
\operatorname{tr}\left(\Theta_{g}(y) \Theta_{g}(y)^{*}\right) & =\operatorname{Tr}\left(p_{o}\right)^{-1} \operatorname{Tr}\left(\Theta_{g}(y) \Theta_{g}(y)^{*} p_{o}\right) \\
& =\operatorname{Tr}\left(p_{o}\right)^{-1} \sum_{s, t \in\left\langle G / G_{o}, g_{o}\right\rangle} \operatorname{Tr}\left(\sigma_{s}(y) \sigma_{t}(y)^{*} p_{o}\right) \\
& =\operatorname{Tr}\left(p_{o}\right)^{-1} \sum_{s \in G_{o} \cap\left\langle G / G_{o, g o}\right\rangle} \operatorname{Tr}\left(\sigma_{s}(y) \sigma_{s}(y)^{*}\right) \text { since } y \in p_{o} S p_{g o} \\
& =\operatorname{Tr}\left(p_{o}\right)^{-1} \sum_{s \in G_{o} \cap\left\langle G / G_{o, g o}\right\rangle} \operatorname{Tr}\left(y y^{*}\right) \text { by (10) } \\
& =\frac{\left[G_{o}: G_{o, g o}\right]}{\operatorname{Tr}\left(p_{o}\right)}\|y\|_{2}^{2} .
\end{aligned}
$$

Hence, $\Theta_{g}$ extends to an injective bounded linear operator from $H_{g}$ to $L^{2}\left(S^{G}, t r\right)$. Observe that $\Theta_{g}$ is a $T^{G}$-bimodular map. Consider $z \in S^{G}$ and $y=p_{o} z p_{g o}$. We have that

$$
\begin{aligned}
\beta \circ \Theta_{g}(y) & =\sum_{t \in\left\langle G_{o} \backslash G / G_{o}\right\rangle} \sum_{s \in\left\langle G / G_{o, g o}\right\rangle} p_{o} \sigma_{s}(y) p_{t o} \sqrt{\frac{\left[G_{o}: G_{o, t o}\right]}{\operatorname{Tr}\left(p_{o}\right)}} \\
& =\sum_{t \in\left\langle G_{o} \backslash G / G_{o}\right\rangle} \sum_{s \in\left\langle G / G_{o, g o}\right\rangle} \delta_{o, s o} \delta_{s g o, t o} p_{o} z p_{t o} \sqrt{\frac{\left[G_{o}: G_{o, t o}\right]}{\operatorname{Tr}\left(p_{o}\right)}},
\end{aligned}
$$

where $\delta_{a, b}$ is the Kronecker symbol. Observe that if $o=s o$ and $s g o=t o$, then $s \in G_{o}$ and $G_{o} g G_{o}=G_{o} t G_{o}$. Therefore,

$$
\begin{aligned}
\beta \circ \Theta_{g}(y) & =\sum_{s \in G_{o} \cap\left\langle G / G_{o, g o}\right\rangle} \delta_{s g o, g o} p_{o} z p_{g o} \sqrt{\frac{\left[G_{o}: G_{o, g o}\right]}{\operatorname{Tr}\left(p_{o}\right)}} \\
& =y \sqrt{\frac{\left[G_{o}: G_{o, g o}\right]}{\operatorname{Tr}\left(p_{o}\right)}}
\end{aligned}
$$

We obtain that $\beta \circ \Theta_{g}=\sqrt{\frac{\left[G_{o}: G_{o, g o}\right]}{\operatorname{Tr}\left(p_{o}\right)}} I d_{H_{g}}$ by a density argument. Therefore, $H_{g}$ is contained in $\operatorname{Im} \beta$ for any $g \in\left\langle G_{o} \backslash G / G_{o}\right\rangle$. Since $\operatorname{Im} \beta \subset \bigoplus_{l \in\left\langle G_{o} \backslash G / G_{o}\right\rangle} H_{l}$ and $\beta$ is an isometry, we 
obtain that $\operatorname{Im} \beta=\bigoplus_{l \in\left\langle G_{o} \backslash G / G_{o}\right\rangle} H_{l}$. Hence, $\beta$ realizes an isomorphism of $T^{G}$-bimodules from $L^{2}\left(S^{G}, t r\right)$ onto $\bigoplus_{l \in\left\langle G_{o} \backslash G / G_{o}\right\rangle} H_{l}$.

Proposition 3.6. Let $f: G \longrightarrow \mathbb{C}$ be a unital bounded $G_{o}$-bi-invariant map. Denote by $\bar{f}: G / G_{o} \longrightarrow \mathbb{C}$ the induced map on the coset space. The following assertions are true.

(1) There exists a unique normal trace-preserving unital T-bimodular map $\phi_{f}: S \longrightarrow$ $S$ such that $\phi_{f}(x)=f\left(h^{-1} g\right) x$ for any $x \in p_{g o} S p_{h o}$ and $g, h \in G$.

(2) If $f$ is positive definite, then $\phi_{f}$ is completely positive.

(3) The restriction of $\phi_{f}$ to $S^{G}$ defines a trace-preserving $T^{G}$-bimodular map $\psi_{f}$ : $S^{G} \longrightarrow S^{G}$.

(4) The map $\bar{f}$ has finite support if and only if $\psi_{f}\left(S^{G}\right)$ is a bifinite $T^{G}$-bimodule.

Proof. Proof of (1). The map $\phi_{f}$ is well defined on the weakly dense $*$-subalgebra $B=$ $\left\{x \in G r \mathcal{P} \otimes G r \mathcal{P}: \exists F \subset\left\langle G / G_{o}\right\rangle\right.$ finite such that $\left.x=\sum_{s, t \in F} p_{s o} x p_{t o}\right\}$. We have that

$$
\begin{aligned}
\operatorname{Tr} \circ \phi_{f}(x) & =\operatorname{Tr}\left(\sum_{g, h \in F} f\left(h^{-1} g\right) p_{g o} x p_{h o}\right)=\sum_{g \in F} \operatorname{Tr}\left(f\left(g^{-1} g\right) p_{g o} x p_{g o}\right) \\
& =\sum_{g \in F} \operatorname{Tr}\left(p_{g o} x p_{g o}\right)=\operatorname{Tr}(x), \text { for any } x \in B .
\end{aligned}
$$

Therefore, $\phi_{f}$ is trace-preserving on $B$. The map $\phi_{f}$ is ultraweakly continuous and extends to a normal trace-preserving map $\phi_{f}: S \longrightarrow S$. This map is clearly unital since $f$ is unital. Consider $t_{1}, t_{2} \in T$ and $x \in \sum_{g, h \in F} p_{g o} S p_{h o}$ where $F$ is a finite set. Observe that $t_{1} p_{g o} x p_{h o} t_{2} \in p_{g o} S p_{h o}$ for any $g, h \in\left\langle G / G_{o}\right\rangle$. Therefore,

$$
\phi_{f}\left(t_{1} x t_{2}\right)=\phi_{f}\left(\sum_{g, h \in F} t_{1} p_{g o} x p_{h o} t_{2}\right)=\sum_{g, h \in F} f\left(h^{-1} g\right) t_{1} p_{g o} x p_{h o} t_{2}=t_{1} \phi_{f}(x) t_{2} .
$$

Hence, by a density argument we have that the map $\phi_{f}$ is $T$-bimodular.

Proof of (2). Suppose that $f$ is positive definite. There exists a Hilbert space $\mathcal{H}$ and a continuous right- $G_{o}$-invariant bounded map $\xi: G \longrightarrow \mathcal{H}$ such that $f\left(h^{-1} g\right)=\langle\xi(h), \xi(g)\rangle$ for any $g, h \in G$. Consider the *-representation $\rho: S \longrightarrow B\left(L^{2}(S, T r) \otimes \mathcal{H}\right)$ defined as $\rho(x)(a \otimes \zeta)=x a \otimes \zeta$ for any $x \in S, a \in L^{2}(S, T r)$ and $\zeta \in \mathcal{H}$. Consider the bounded operator $A: L^{2}(S, T r) \longrightarrow L^{2}(S, T r) \otimes \mathcal{H}, a \in p_{g o} L^{2}(S, T r) \longmapsto a \otimes \xi(g)$, for any $g \in\left\langle G / G_{o}\right\rangle$. One can check that $\phi_{f}(x)=A^{*} \rho(x) A$ for any $x \in S$. Therefore, $\phi_{f}$ is completely positive.

Proof of (3). Consider the action $\sigma: G \longrightarrow \operatorname{Aut}(S)$. Since $\phi_{f}$ does not depend on the choice of system of representatives $\left\langle G / G_{o}\right\rangle$ we get that $\phi_{f} \circ \sigma_{g}=\sigma_{g} \circ \phi_{f}$ for any $g \in G$. This implies that $\phi_{f}\left(S^{G}\right)$ is contained inside $S^{G}$. The map $\psi_{f}$ is $T^{G}$-bimodular since it is the restriction of a $T$-bimodular map. Consider the normal map $E_{T}^{S}: S \longrightarrow S, x \longmapsto$ $\sum_{g \in\left\langle G / G_{o}\right\rangle} p_{g o} x p_{g o}$. The unique normal faithful tracial state $\operatorname{tr}$ of $S^{G}$ is the restriction of the map $\omega: S \longrightarrow \mathbb{C}, x \longmapsto \operatorname{Tr}\left(p_{o}\right)^{-1} \operatorname{Tr}\left(x p_{o}\right)$. It is easy to see that $\omega \circ E_{T}^{S}=\omega$ and $E_{T}^{S} \circ \phi_{f}=E_{T}^{S}$. Therefore,

$$
\operatorname{tr} \circ \psi_{f}(x)=\omega \circ \phi_{f}(x)=\omega \circ E_{T}^{S} \circ \phi_{f}(x)=\omega(x)=\operatorname{tr}(x),
$$

for any $x \in S^{G}$.

Proof of (4). Since $G / G_{o}$ is an almost normal subgroup, we have that $\bar{f}$ is finitely supported if and only if the support $\operatorname{supp}(f)$ is contained in finitely many double cosets of $G_{o} \backslash G / G_{o}$. Since $f$ is $G_{o}$-bi-invariant, we have that $\operatorname{supp}(f)$ is stable by left and right 
multiplication by $G_{o}$. Hence, there exists $E \subset\left\langle G_{o} \backslash G / G_{o}\right\rangle$ such that $\operatorname{supp}(f)=G_{o} \cdot E \cdot G_{o}$. Observe that the norm closure of the image of $\psi_{f}$ in $L^{2}\left(S^{G}, t r\right)$ is isomorphic to $\bigoplus_{g \in E} H_{g}$. Proposition 3.5 implies the result.

Proof of Theorem $A$. Consider $(\Gamma, \mu), G, G_{o}, \mathcal{P}$ as above such that $G$ is amenable. Denote by $\mathcal{Q}=\mathcal{P}^{G}$ the subfactor planar algebra equal to the fixed point space under the action of $G$. As observed in Section 1.3, the subgroup $G_{o}<G$ is co-amenable since $G$ is amenable. By Section 1.3, there exists a sequence of positive definite $G_{o^{-}}$-bi-invariant maps $f_{n}: G \longrightarrow \mathbb{C}, n \geqslant 0$ with support contained in a finite union of right $G_{o^{-}}$-cosets and that converges pointwise to 1 . By Proposition 3.6, the collection $\left(\psi_{n}:=\psi_{f_{n}}, n \geqslant 0\right)$ is a sequence of completely positive $T^{G}$-bimodular maps from $S^{G}$ to $S^{G}$ such that $\psi_{n}\left(S^{G}\right)$ is a bifinite $T^{G}$-bimodule for any $n \geqslant 0$. It is easy to see that $\lim _{n \rightarrow \infty}\left\|\psi_{n}(x)-x\right\|_{2}=0$ for any $x \in S^{G}$. Therefore, $T^{G} \subset S^{G}$ is co-amenable.

Consider the inclusion $M^{G} \vee\left(M^{\text {op }}\right)^{G} \subset T^{G}$. The map $\Theta: T(o)^{G_{o}} \longrightarrow S, x \longmapsto$ $\sum_{s \in\left\langle G / G_{o}\right\rangle} \sigma_{s}(x)$ realizes an isomorphism from $T(o)^{G_{o}}$ onto $T^{G}$ such that $\Theta\left(M(o)^{G_{o}}\right)=M^{G}$ and $\Theta\left(\left(M(o)^{\mathrm{op}}\right)^{G_{o}}\right)=\left(M^{\mathrm{op}}\right)^{G}$. Therefore, the inclusion $M^{G} \vee\left(M^{\mathrm{op}}\right)^{G} \subset T^{G}$ is isomorphic to $T(o)^{G_{o} \times G_{o}} \subset T(o)^{G_{o}}$ for the action $\sigma \otimes \sigma^{\mathrm{op}}: G_{o} \times G_{o} \longrightarrow \operatorname{Aut}(M(o)) \times \operatorname{Aut}\left(M(o)^{\mathrm{op}}\right)<$ Aut $(T(o))$. The group $G_{o}$ is amenable since it is a closed subgroup of the amenable group $G$. By the same observation made in Section 1.3, we have that the subgroup $G_{o} \subset G_{o} \times G_{o}$ given by the diagonal inclusion is co-amenable since $G_{o} \times G_{o}$ is amenable. Therefore, the inclusion $M^{G} \vee\left(M^{\text {op }}\right)^{G} \subset T^{G}$ is co-amenable by [20, Proposition 6]. The composition of two co-amenable inclusions is co-amenable by [25, Theorem 3.2.4.1]. Therefore, $M^{G} \vee\left(M^{\text {op }}\right)^{G} \subset S^{G}$ is co-amenable. Proposition 3.3 implies that this later inclusion is isomorphic to the symmetric enveloping inclusion of $\mathcal{Q}$. Therefore, the subfactor planar algebra $\mathcal{Q}$ is amenable.

We end this section with an observation regarding the principal graph of $\mathcal{Q}$.

Proposition 3.7. Let $(\Gamma, \mu), G, G_{o}$, and $\mathcal{Q}$ satisfying the assumptions of this section. Moreover, assume that $G$ is contained in $A u t(\Gamma, \mu)$. Then the subfactor planar algebra $\mathcal{Q}$ has finite depth if and only if the graph $\Gamma$ is finite.

Proof. Recall that a subfactor planar algebra $\mathcal{Q}$ has finite depth if and only if its symmetric enveloping inclusion is a finite index subfactor. The symmetric enveloping inclusion of $\mathcal{Q}$ is isomorphic to $M^{G} \vee\left(M^{\mathrm{op}}\right)^{G} \subset S^{G}$ by Proposition 3.3. By Proposition 3.5, the space $L^{2}\left(S^{G}, t r\right)$ is isomorphic to a sum of bifinite $T^{G}$-bimodules indexed by $G_{o} \backslash G / G_{o}$.

Suppose that $\Gamma$ is infinite. Observe that $f: G \longrightarrow \mathbb{N}, g \longmapsto d(o, g o)$ is $G_{o}$-bi-invariant map. Moreover, $\operatorname{Imf}=\left\{m \in \mathbb{N}: \exists w \in V^{+}, d(o, w)=m\right\}$ since $G$ acts transitively on $V^{+}$. Therefore, $\left|G_{o} \backslash G / G_{o}\right| \geqslant|\operatorname{Im} f|$. Since $\Gamma$ has infinitely many vertices and is locally finite, there exists vertices in $V^{+}$that are arbitrary far from $o \in V^{+}$. Hence, $\operatorname{Imf}$ is infinite and $\left|G_{o} \backslash G / G_{o}\right|=\infty$. This implies that $\left[S^{G}: M^{G} \vee\left(M^{\mathrm{op}}\right)^{G}\right]=\left[S^{G}: T^{G}\right]\left[T^{G}: M^{G} \vee\left(M^{\mathrm{op}}\right)^{G}\right]=$ $\infty$.

Suppose that $\Gamma$ is finite. Then $\left|G_{o} \backslash G / G_{o}\right| \leqslant\left|G / G_{o}\right|=\left|V^{+}\right|<\infty$. Hence, $\left[S^{G}: T^{G}\right]<$ $\infty$. The group $G_{o}$ is necessarily finite since $\Gamma$ has a finite number of vertices and edges and $G_{o}$ is contained in $\operatorname{Aut}(\Gamma)_{o}$. As observed in the last proof, the inclusion $M^{G} \vee\left(M^{\mathrm{op}}\right)^{G} \subset T^{G}$ is isomorphic to $T(o)^{G_{o} \times G_{o}} \subset T(o)^{G_{o}}$ and $T(o)$ is a factor. Hence, $\left[S^{G}: M^{G} \vee\left(M^{\mathrm{op}}\right)^{G}\right]=$ $\left[S^{G}: T^{G}\right]\left[T^{G}: M^{G} \vee\left(M^{\mathrm{op}}\right)^{G}\right]=\left[S^{G}: T^{G}\right]\left[T(o)^{G_{o}}: T(o)^{G_{o} \times G_{o}}\right]=\left[S^{G}: T^{G}\right]\left|G_{o}\right|<\infty$. This concludes the proof. 


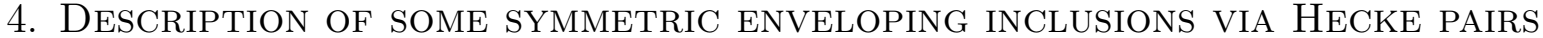

\subsection{Crossed products and Hecke pairs.}

4.1.1. The ordinary action case. Consider an inclusion of groups $H<G$ and assume that $G / H$ is countable. We say that $(G, H)$ is a Hecke pair if the subgroup $H<G$ is almost normal, i.e. for any $g \in G$ the group $H \cap g H^{-1}$ has finite index inside $H$ and $g \mathrm{Hg}^{-1}$. We refer the reader to [1] for more details on Hecke pairs and operator algebras. To $(G, H)$ can be associated a von Neumann algebra $L(G, H)$ analogous to the group von Neumann algebra. In this section, we define the cocycle action of a Hecke pair on a tracial von Neumann algebra and its corresponding twisted crossed product. This has been considered in the framework of ordinary action on $C^{*}$-algebras by Palma, see [23, 24]. Our approach is a natural generalization of the construction given in [1, Section 1]. We first define this notion in the ordinary action case which has much simpler formulas.

Let $(A, \tau)$ be a finite von Neumann algebra with a normal faithful tracial state. Let $\gamma: G \longrightarrow \operatorname{Aut}(A, \tau)$ be a trace-preserving action of $G$ on $A$. Let $\mathbb{C}[A ; G, H]$ be the space of functions $f: G \longrightarrow A$ such that $f(h g k)=\gamma_{h}(f(g))$ for any $g \in G, h, k \in H$, and such that the induced functions $\bar{f}: G / H \longrightarrow A$ is finitely supported. Note that if $f \in \mathbb{C}[A ; G, H]$ and $g \in G$, then $f(g)$ is fixed by $\sigma\left(H \cap g H g^{-1}\right)$. We define a multiplication and an involution $*$ on $\mathbb{C}[A ; G, H]$ as follows.

$$
f_{1} f_{2}(g)=\sum_{s \in\langle G / H\rangle} f_{1}(s) \gamma_{s}\left(f_{2}\left(s^{-1} g\right)\right) \text {, for any } f_{1}, f_{2} \in \mathbb{C}[A ; G, H] \text { and } g \in G,
$$

where $\langle G / H\rangle$ is a system of representatives of $G / H$,

$$
f^{*}(g)=\gamma_{g}\left(f\left(g^{-1}\right)^{*}\right), \text { for any } f \in \mathbb{C}[A ; G, H] \text { and } g \in G .
$$

The space $\mathbb{C}[A ; G, H]$ endowed with those operations is a unital *-algebra. We have an inclusion of $A^{H}$ inside $\mathbb{C}[A ; G, H]$ given by the map $j: a \in A^{H} \longmapsto f_{a} \in \mathbb{C}[A ; G, H]$ such that $f_{a}(g)=a$ if $g \in H$ and zero otherwise. We identify $A^{H}$ and $j\left(A^{H}\right)$. Consider the linear functional $\omega: \mathbb{C}[A ; G, H] \longrightarrow \mathbb{C}, f \longmapsto \tau(f(1))$. Let $\pi=\gamma \otimes \operatorname{Ad}(\lambda): G \longrightarrow$ $\operatorname{Aut}\left(A \bar{\otimes} B\left(\ell^{2}(G / H)\right)\right)$ be the group action defined as $\pi_{g}\left(a \otimes e_{h, k}\right)=\gamma_{g}(a) \otimes e_{g h, g k}$, for any

$g \in G, h, k \in G / H$ and $a \in A$. Denote by $\operatorname{Tr}: B\left(\ell^{2}(G / H)\right)_{+} \longrightarrow \overline{\mathbb{R}}_{+}$the usual normal faithful semi-finite tracial weight on $B\left(\ell^{2}(G / H)\right)$ that sends any minimal projection to 1 .

Proposition 4.1. The linear functional $\omega$ is faithful and the *-algebra $\mathbb{C}[A ; G, H]$ acts by bounded operators on $L^{2}(\mathbb{C}[A ; G, H], \omega)$ by left multiplication. Let $v N[A ; G, H]$ be the von Neumann algebra generated by this left action that we call the crossed product of $A$ by $(G, H)$. We have an isomorphism $\varphi$ of von Neumann algebras from $v N[A ; G, H]$ onto the fixed point space $\left(A \bar{\otimes} B\left(\ell^{2}(G / H)\right)\right)^{G}$ such that $\varphi(f)=\sum_{s, t \in\langle G / H\rangle} \gamma_{s}(f(t)) \otimes e_{s, s t}$ for any $f \in \mathbb{C}[A ; G, H]$. In particular,

$$
\varphi\left(A^{H}\right)=\left\{\sum_{s \in\langle G / H\rangle} \gamma_{s}(a) \otimes e_{s, s}: a \in A^{H}\right\} .
$$

Proof. If $f \in \mathbb{C}[A ; G, H]$, then $\omega\left(f f^{*}\right)=\tau\left(f f^{*}(1)\right)=\sum_{s \in\langle G / H\rangle} \tau\left(f(s) f(s)^{*}\right)$. Hence, if $\omega\left(f f^{*}\right)=0$, then $f(s)=0$ for any $s \in\langle G / H\rangle$ since $\tau$ is faithful. This implies that $\omega$ is faithful.

Consider the Hilbert space $\ell^{2}(G / H, A)$ of right- $H$-invariant functions $f: G \longrightarrow L^{2}(A)$ such that $\sum_{s \in\langle G / H\rangle} \tau\left(f(s) f(s)^{*}\right)<\infty$ with the norm $\|f\|_{2}=\sqrt{\sum_{s \in\langle G / H\rangle} \tau\left(f(s) f(s)^{*}\right)}$. 
The Hilbert space $L^{2}(\mathbb{C}[A ; G, H], \omega)$ is isometric to a subspace of $\ell^{2}(G / H, A)$. We identify $L^{2}(\mathbb{C}[A ; G, H], \omega)$ and this isometric subspace. Consider $r \in\langle G / H\rangle$ and $\left\{h_{1}, \cdots, h_{k}\right\} \subset$ $H$ such that $H r H$ is equal to the disjoint union of the right cosets $\bigcup_{i=1}^{k} h_{i} r H$. Consider $a \in A$ and the function $f \in \mathbb{C}[A ; G, H]$ such that its support is contained in $\mathrm{HrH}$ and $f(r)=a$. Consider an element $\xi \in \mathbb{C}[A ; G, H] \subset L^{2}(\mathbb{C}[A ; G, H], \omega)$. We have that

$$
(f \xi)(g)=\sum_{s \in\langle G / H\rangle} f(s) \gamma_{s}\left(\xi\left(s^{-1} g\right)\right)=\sum_{i=1}^{k} \gamma_{h_{i}}(a) \gamma_{h_{i} r}\left(\xi\left(\left(h_{i} r\right)^{-1} g\right)\right), g \in G .
$$

We fix $1 \leqslant i \leqslant k$. Consider the map $\eta_{i}: g \in G \longmapsto \gamma_{h_{i}}(a) \gamma_{h_{i} r}\left(\xi\left(\left(h_{i} r\right)^{-1} g\right)\right)$. Note that $\eta_{i} \in \ell^{2}(G / H, A)$ and $f \xi=\sum_{j=1}^{k} \eta_{j}$. We have that

$$
\begin{aligned}
\left\|\eta_{i}\right\|_{2}^{2} & =\sum_{s \in\langle G / H\rangle} \tau\left(\gamma _ { h _ { i } } ( a ) \gamma _ { h _ { i } r } \left(\xi\left(\left(h_{i} r\right)^{-1} s\right) \gamma_{h_{i} r}\left(\xi\left(\left(h_{i} r\right)^{-1} s\right)^{*} \gamma_{h_{i}}(a)^{*}\right)\right.\right. \\
& =\sum_{s \in\langle G / H\rangle} \tau\left(a^{*} a \gamma_{r}\left(\xi\left(\left(h_{i} r\right)^{-1} s\right) \xi\left(\left(h_{i} r\right)^{-1} s\right)^{*}\right)\right) \\
& \leqslant\|a\|_{A}^{2} \sum_{s \in\langle G / H\rangle} \tau\left(\xi\left(\left(h_{i} r\right)^{-1} s\right) \xi\left(\left(h_{i} r\right)^{-1} s\right)^{*}\right)=\|a\|_{A}^{2}\|\xi\|_{2}^{2},
\end{aligned}
$$

where $\|\cdot\|_{A}$ is the $\mathrm{C}^{*}$-norm of $A$. Hence, $\|f \xi\|_{2} \leqslant k\|a\|_{A}\|\xi\|_{2}$. Since the subspace $\mathbb{C}[A ; G, H] \subset L^{2}(\mathbb{C}[A ; G, H], \omega)$ is dense, we obtain that the left multiplication by $f$ on $L^{2}(\mathbb{C}[A ; G, H], \omega)$ is bounded. Therefore, the left multiplication of $\mathbb{C}[A ; G, H]$ defines a $*$-representation of $\mathbb{C}[A ; G, H]$ on the GNS Hilbert space $L^{2}(\mathbb{C}[A ; G, H], \omega)$. It is clear the $\varphi$ defines a normal *-morphism from $\mathrm{vN}[A ; G, H]$ to the fixed point space $\left(A \bar{\otimes} B\left(\ell^{2}(G / H)\right)\right)^{G}$. If $\varphi(f)=0$, then $\gamma_{s}(f(t))=0$ for any $s, t \in\langle G / H\rangle$. Hence, $f=0$ and $\varphi$ is injective. Consider $x=\sum_{s, t \in G / H} x_{s, t} \otimes e_{s, t} \in\left(A \bar{\otimes} B\left(\ell^{2}(G / H)\right)\right)^{G}$. Since $x$ is $G$-invariant, we have that $x_{g s, g t}=\gamma_{g}\left(x_{s, t}\right)$ for any $g \in G, s, t \in G / H$. Therefore, $x=\varphi(f)$ where $f(t)=x_{1, t}, t \in\langle G / H\rangle$. Hence, $\varphi$ is surjective. We obtain that $\varphi$ realizes an isomorphism of von Neumann algebras. The last assertion of the proposition follows from the definition of $\varphi$.

4.1.2. The twisted case. Let $G, H, A, \tau$ be as above. A cocycle action of the Hecke pair $(G, H)$ on the tracial von Neumann algebra $(A, \tau)$ is a couple of maps $(\gamma, u)$ where

$$
\gamma: G \times G / H \longrightarrow \operatorname{Aut}(A, \tau) \text { and } u: G \times G / H \times G / H \longrightarrow U(A)
$$

satisfying the following axioms:

(1) $\gamma_{1, s}=\mathrm{Id}$,

(2) $\gamma_{g h, s}=\gamma_{g, h s} \circ \gamma_{h, s}$,

(3) $\gamma_{g, s}=\operatorname{Ad}\left(u_{g, s, t}\right) \circ \gamma_{g, t}$,

(4) $u_{1, s, t}=u_{g, s, s}=1$,

(5) $u_{g, s, t} u_{g, t, r}=u_{g, s, r}$, and

(6) $u_{g h, s, t}=\gamma_{g, h s}\left(u_{h, s, t}\right) u_{g, h s, h t}$ for any $g, h \in G$ and $s, t, r \in G / H$.

We continue to denote by $\mathbb{C}[A ; G, H]$ the space of functions $f: G \longrightarrow A$ such that $f(h g k)=\gamma_{h, 1}(f(g)) u_{h, 1, g}$ for any $g \in G, h, k \in H$, and such that the induced functions $\bar{f}: G / H \longrightarrow A$ is finitely supported. We define a multiplication and an involution $*$ on 
$\mathbb{C}[A ; G, H]$ as follows.

$$
f_{1} f_{2}(g)=\sum_{s \in\langle G / H\rangle} f_{1}(s) \gamma_{s, 1}\left(f_{2}\left(s^{-1} g\right)\right) u_{s, 1, s^{-1} g} \text {, for any } f_{1}, f_{2} \in \mathbb{C}[A ; G, H] \text { and } g \in G,
$$

where $\langle G / H\rangle$ is a system of representatives of $G / H$,

$$
f^{*}(g)=\gamma_{g, g^{-1}}\left(f\left(g^{-1}\right)^{*}\right) u_{g, g^{-1}, 1}, \text { for any } f \in \mathbb{C}[A ; G, H] \text { and } g \in G .
$$

A careful check shows that the space $\mathbb{C}[A ; G, H]$ endowed with those operations is a unital associative $*$-algebra. Observe that the map $h \in H \longmapsto \gamma_{h, 1} \in \operatorname{Aut}(A, \tau)$ is a group morphism. Hence, we have an ordinary action of $H$ on $A$. Denote by $A^{H}$ the algebra of $a \in A$ such that $\gamma_{h, 1}(a)=a$ for any $h \in H$. We have an inclusion of $A^{H}$ inside $\mathbb{C}[A ; G, H]$ given by the map $j: a \in A^{H} \longmapsto f_{a} \in \mathbb{C}[A ; G, H]$ such that $f_{a}(g)=a$ if $g \in H$ and zero otherwise. Consider the linear functional $\omega: \mathbb{C}[A ; G, H] \longrightarrow \mathbb{C}, f \longmapsto \tau(f(1))$.

Proposition 4.2. The linear functional $\omega$ is faithful and the *-algebra $\mathbb{C}[A ; G, H]$ acts by bounded operators on $L^{2}(\mathbb{C}[A ; G, H], \omega)$ by left multiplication. Let $v N[A ; G, H]$ be the von Neumann algebra generated by this left action that we call the twisted crossed product of $A$ by $(G, H)$. There exists a unique group morphism $\pi: G \longrightarrow A u t\left(A \bar{\otimes} B\left(\ell^{2}(G / H)\right)\right)$ satisfying that $\pi_{g}\left(a \otimes e_{s, t}\right)=\gamma_{g, s}(a) u_{g, s, t} \otimes e_{g s, g t}$ for any $a \in A, g \in G, s, t \in G / H$. We have an isomorphism $\varphi$ of von Neumann algebras from $v N[A ; G, H]$ onto the fixed point space $\left(A \bar{\otimes} B\left(\ell^{2}(G / H)\right)\right)^{G}$ such that $\varphi(f)=\sum_{s, t \in\langle G / H\rangle} \gamma_{s, 1}(f(t)) u_{s, 1, t} \otimes e_{s, s t}$ for any $f \in \mathbb{C}[A ; G, H]$. In particular,

$$
\varphi\left(A^{H}\right)=\left\{\sum_{s \in\langle G / H\rangle} \gamma_{s, 1}(a) \otimes e_{s, s}: a \in A^{H}\right\} .
$$

Proof. The proof is similar to the proof of Proposition 4.1.

Remark 4.3. If $A=\mathbb{C}$, then the von Neumann algebra vN $[A ; G, H]$ is the von Neumann algebra $L(G, H)$ (possibly twisted by a cocycle) associated to the Hecke pair $(G, H)[1$. If $H<G$ is a normal subgroup, then the von Neumann algebra vN $[A ; G, H]$ is isomorphic to a (twisted) crossed product $A^{H} \rtimes(G / H)$ of the fixed point space $A^{H}$ by the quotient group $G / H$. If the standard system of matrix units of $B\left(\ell^{2}(G / H)\right)$ is $G$-invariant, i.e. $\pi_{g}\left(1 \otimes e_{s, t}\right)=1 \otimes e_{g s, g t}$ for any $g \in G, s, t \in G / H$, then $\left(A \bar{\otimes} B\left(\ell^{2}(G / H)\right)\right)^{G}$ is isomorphic to $\mathrm{vN}[A ; G, H]$ for an ordinary action of $(G, H)$ on $A$.

4.2. Description of symmetric enveloping inclusions. Let $(\Gamma, \mu)$ be a weighted graph with vertex set $V=V^{+} \cup V^{+}$and let $\mathcal{P}$ be its associated bipartite graph planar algebra. Moreover, assume that $\mu$ is constant on the set of edges with source in $V^{+}$. Let us fix a vertex $o \in V^{+}$and denote by $T \subset S$ the symmetric enveloping inclusion of $\mathcal{P}$. The set $\left\{e_{v}: v \in V^{+}\right\}$is the set of minimal projections of $\ell^{\infty}\left(V^{+}\right)$and $\left\{e_{v, w}: v, w \in V^{+}\right\}$ is the standard system of matrix units of $B\left(\ell^{2}\left(V^{+}\right)\right)$. We continue to denote by $T(v)$ the corner $T p_{v}$ for any $v \in V^{+}$.

Theorem 4.4. The symmetric enveloping inclusion of $\mathcal{P}$ is isomorphic to

$$
T(o) \bar{\otimes} \ell^{\infty}\left(V^{+}\right) \subset T(o) \bar{\otimes} B\left(\ell^{2}\left(V^{+}\right)\right),
$$

where the inclusion is given by $a \otimes e_{v} \mapsto a \otimes e_{v, v}$ for any $a \in T(o), v \in V^{+}$. 
Proof. By Proposition 2.2. $\operatorname{Tr}\left(p_{v}\right)=\operatorname{Tr}\left(p_{o}\right)$ for any $v \in V^{+}$. Since $S$ is a factor, there exists a system of matrix units $\left\{\epsilon_{v, w}: v, w \in V^{+}\right\} \subset S$ such that $\epsilon_{v, w} \epsilon_{v, w}^{*}=p_{v}$ and $\epsilon_{v, w}^{*} \epsilon_{v, w}=p_{w}$ for any $v, w \in V^{+}$. Consider $v \in V^{+}$and the map $\beta_{v}: T(o) \longrightarrow S, x \longmapsto$ $\epsilon_{v, o} x \epsilon_{o, v}$. Since $p_{v} S p_{v}=T(v)$, we have that the range of $\beta_{v}$ is contained in $T(v)$. Observe that $\beta_{v}$ realizes an isomorphism of von Neumann algebras from $T(o)$ onto $T(v)$ and its inverse is the map $\beta_{v}^{-1}: T(v) \longrightarrow T(o), x \longmapsto \epsilon_{o, v} x \epsilon_{v, o}$. Observe that $S$ is the weak closure of the vector $\operatorname{space} \operatorname{Span}\left(x \epsilon_{v, w}: v, w \in V^{+}, x \in T(v)\right)$. Consider the densely defined map $\phi: S \longrightarrow T(o) \bar{\otimes} B\left(\ell^{2}\left(V^{+}\right)\right)$defined as $\phi\left(x \epsilon_{v, w}\right)=\beta_{v}^{-1}(x) \otimes e_{v, w}$ for any $v, w \in V^{+}, x \in T(v)$. This map is clearly a $*$-morphism and extends to a normal map from $S$ to $T(o) \bar{\otimes} B\left(\ell^{2}\left(V^{+}\right)\right)$. Since $S$ is a factor and $\phi$ is not identically equal to zero we have that $\phi$ is injective. The range of $\phi$ is weakly dense since it contains the set $\left\{x \otimes e_{v, w}: x \in T(o), v, w \in V^{+}\right\}$. This implies that $\phi$ is surjective. Therefore, $\phi$ realizes an isomorphism of von Neumann algebras. If $x \in T$, then $\phi(x)=\sum_{v \in V^{+}} \beta_{v}^{-1}\left(x p_{v}\right) \otimes e_{v, v}$. This implies that $\phi(T)$ is contained in $T(o) \bar{\otimes} \ell^{\infty}\left(V^{+}\right)$. Furthermore, $\phi(T)$ is clearly dense in $T(o) \bar{\otimes} \ell^{\infty}\left(V^{+}\right)$. Therefore, $\phi(T)=T(o) \bar{\otimes} \ell^{\infty}\left(V^{+}\right)$.

Consider a subgroup $G<\operatorname{Aut}(\mathcal{P})$ such that $\mathcal{Q}:=\mathcal{P}^{G}$ is a subfactor planar algebra. In particular, $G$ acts transitively on $V^{+}$. We identify $V^{+}$and $G / G_{0}$. Let $\sigma: G \longrightarrow \operatorname{Aut}(S)$ be the action defined in Section 3. Note that we have an action of $G_{o} \times G_{o}$ on the corner $T(o)$ given by $(g, h) \cdot a \otimes b^{\text {op }}=\sigma_{g}(a) \otimes \sigma_{h}(b)^{\text {op }}$ for any $g, h \in G_{o}, a, b \in M(o)$. We denote by $T(o)^{G_{o} \times G_{o}}$ the fixed point space with respect to this action. Consider the diagonal inclusion of groups $g \in G_{o} \longmapsto(g, g) \in G_{o} \times G_{o}$ and the corresponding inclusion of von Neumann algebras $T(o)^{G_{o} \times G_{o}} \subset T(o)^{G_{o}}$. Denote by $\mathcal{Q}=\mathcal{P}^{G}$ the fixed point space of $\mathcal{P}$ with respect to the action of $G$.

Theorem 4.5. There exists a cocycle action $(\gamma, u)$ of the Hecke pair $\left(G, G_{o}\right)$ on the $I I_{1}$ factor $T(o)$ such that $\gamma_{g, 1}\left(a \otimes b^{o p}\right)=\sigma_{g}(a) \otimes \sigma_{g}(b)^{o p}$ for any $g \in G, a, b \in M(o)$, and such that the symmetric enveloping inclusion of $\mathcal{Q}$ is isomorphic to

$$
T(o)^{G_{o} \times G_{o}} \subset v N\left[T(o) ; G, G_{o}\right] .
$$

Proof. The map $g \in G \longmapsto g o \in V^{+}$is surjective since the action is transitive. It realizes a bijection from $G / G_{o}$ onto $V^{+}$. Denote by $\left\langle G / G_{o}\right\rangle$ a system of representatives of $G / G_{o}$. By Theorem 4.4, the symmetric enveloping inclusion of $\mathcal{P}$ is isomorphic to $T(o) \bar{\otimes} \ell^{\infty}\left(V^{+}\right) \subset T(o) \bar{\otimes} B\left(\ell^{2}\left(V^{+}\right)\right)$via the map $\phi: S \longrightarrow T(o) \bar{\otimes} B\left(\ell^{2}\left(V^{+}\right)\right)$that satisfies $\phi\left(a \epsilon_{v, w}\right)=\epsilon_{o, v} a \epsilon_{v, o} \otimes e_{v, w}$ for any $v, w \in V^{+}, a \in T(v)$. We have that the group action

$$
\pi: G \longrightarrow \operatorname{Aut}\left(T(o) \bar{\otimes} B\left(\ell^{2}\left(V^{+}\right)\right), g \longmapsto \phi \circ \sigma_{g} \circ \phi^{-1}\right.
$$

satisfies that

$$
\pi_{g}\left(T(o) \otimes e_{v, w}\right)=T(o) \otimes e_{g v, g w} \text { and } \pi_{g}\left(1 \otimes e_{v, v}\right)=1 \otimes e_{g v, g v} \text { for any } g \in G, v, w \in V^{+} .
$$

This implies that for any $g \in G, v, w \in V^{+}$there exists a unitary $u_{g, v, w} \in U(T(o))$ such that $\pi_{g}\left(1 \otimes e_{v, w}\right)=u_{g, v, w} \otimes e_{g v, g w}$. Furthermore, for any $a \in T(o), v \in V^{+}, g \in G$ there exists $\gamma_{g, v}(a) \in T(o)$ such that $\pi_{g}\left(a \otimes e_{v, v}\right)=\gamma_{g, v}(a) \otimes e_{g v, g v}$. Since $\pi_{g}$ is an automorphism and $\pi_{g}\left(T(o) \otimes e_{v, v}\right)=T(o) \otimes e_{g v, g v}$ we necessarily have that $\gamma_{g, v}$ is an automorphism of $T(o)$ for any $g \in G, v \in V^{+}$. From (11) and the fact that $\pi$ is a group action we can deduce that $(\gamma, u)$ is a cocycle action of $\left(G, G_{o}\right)$ on the $\mathrm{II}_{1}$ factor $T(o)$. Let $\operatorname{vN}\left[T(o) ; G, G_{o}\right]$ be the crossed product von Neumann algebra associated to the cocycle action $(\gamma, u)$ of the 
Hecke pair $\left(G, G_{o}\right)$ on the $\mathrm{II}_{1}$ factor $T(o)$. By Proposition 4.2, we obtain that the inclusion $T(o)^{G_{o}} \subset \mathrm{vN}\left[T(o) ; G, G_{o}\right]$ is isomorphic to the inclusion

$$
\left\{\sum_{s \in\left\langle G / G_{o}\right\rangle} \sigma_{s}(x) \otimes e_{s o, s o}: x \in T(o)^{G_{o}}\right\} \subset\left(T(o) \bar{\otimes} B\left(\ell^{2}\left(V^{+}\right)\right)^{G},\right.
$$

where $\left(T(o) \bar{\otimes} B\left(\ell^{2}\left(V^{+}\right)\right)^{G}\right.$ is the fixed point space under the action of $\pi$. Consider the isomorphism $\phi: S \longrightarrow T(o) \bar{\otimes} B\left(\ell^{2}\left(V^{+}\right)\right.$of the proof of Theorem 4.4. The symmetric enveloping inclusion of $\mathcal{Q}$ is isomorphic to $M^{G} \vee\left(M^{\mathrm{op}}\right)^{G} \subset S^{G}$ by Proposition 3.3. We have that $\phi\left(S^{G}\right)=\left(T(o) \bar{\otimes} B\left(\ell^{2}\left(V^{+}\right)\right)^{G}\right.$. Observe that $\phi\left(M^{G}\right)=\left\{\sum_{s \in\left\langle G / G_{o}\right\rangle} \sigma_{s}(a) \otimes 1 \otimes\right.$ $\left.e_{s o, s o}: a \in M(o)^{G_{o}}\right\}$ and $\phi\left(\left(M^{\mathrm{op}}\right)^{G}\right)=\left\{\sum_{s \in\left\langle G / G_{o}\right\rangle} 1 \otimes\left(\sigma_{s}(b)\right)^{\mathrm{op}} \otimes e_{s o, s o}: b \in M(o)^{G_{o}}\right\}$. This last observation together with the characterization of the image of $T(o)^{G_{o}}$ given in (12) imply that the symmetric enveloping inclusion of $\mathcal{Q}$ is isomorphic to the inclusion $T(o)^{G_{o} \times G_{o}} \subset \mathrm{vN}\left[T(o) ; G, G_{o}\right]$.

4.3. Examples. We present examples of subfactor planar algebras for which we can describe their symmetric enveloping inclusion via our last theorem.

4.3.1. Diagonal subfactors. The symmetric enveloping inclusion of a diagonal subfactor is known to be isomorphic to the (twisted) crossed product of a $\mathrm{II}_{1}$ factor by a group [30, Section 3]. We give here a new proof of this fact which is a particular case of Theorem 4.5. Consider a $\mathrm{II}_{1}$ factor $L$ and a finite set $\left\{g_{1}, \cdots, g_{n}\right\}$ of outer automorphisms of $L$. Denote by $G$ the subgroup of the outer automorphism group $\operatorname{Out}(L)$ generated by $\left\{g_{1}, \cdots, g_{n}\right\}$. Let $N \subset M$ be the subfactor equal to the inclusion $\left\{\sum_{i=1}^{n+1} \alpha_{g_{i}}(x) \otimes e_{i, i}\right.$ : $x \in L\} \subset L \otimes \mathcal{M}_{n+1}(\mathbb{C})$, where $\left\{e_{i, j}: i, j=1, \cdots n+1\right\}$ is the usual system of matrix units of the type $\mathrm{I}_{n+1}$ factor $\mathcal{M}_{n+1}(\mathbb{C})$ and $g_{n+1}=1$. This subfactor is called a diagonal subfactor. By [3, 7], the subfactor planar algebra of $N \subset M$ can be described as the fixed point planar algebra of a bipartite graph planar algebra as follows. Consider the bipartite graph $\Gamma$, where $V^{+}=\left\{v_{g}^{+}: g \in G\right\}$ is a copy of $G$ and $V^{-}=\left\{v_{g}^{-}: g \in G\right\}$ is another copy of $G$. For any $g \in G$ and $1 \leqslant i \leqslant n+1$ there is an edge from $v_{g}^{+}$to $v_{g g_{i}}^{-}$. Let $\mu$ be the weight of $\Gamma$ that assigns 1 to any edge and let $\mathcal{P}$ be the bipartite graph planar algebra associated to $(\Gamma, \mu)$. The group $G$ acts on the weighted graph $(\Gamma, \mu)$ by left multiplication. The fixed point planar algebra $\mathcal{Q}=\mathcal{P}^{G}$ is isomorphic to the subfactor planar algebra of $N \subset M$. For any even vertex of $\Gamma$, the subgroup of $G$ that fixes this vertex is trivial. Therefore, by Theorem 4.5 and Remark 4.3, there exists a $\mathrm{II}_{1}$ factor $A$ and a trace-preserving cocycle action of $G$ on $A$ such that the symmetric enveloping inclusion of $\mathcal{Q}$ is isomorphic to $A \subset A \rtimes G$.

4.3.2. Bisch-Haagerup subfactors. Consider a $\mathrm{II}_{1}$ factor $L$ and two finite subgroups $H, K \subset$ Out $(L)$. Assume that the intersection $H \cap K$ is the trivial group. Denote by $G$ the subgroup of $\operatorname{Out}(L)$ generated by $H$ and $K$. Assume that there exists a group morphism $\alpha: G \longrightarrow A u t(L)$ which is a lift of the inclusion $G \subset O u t(L)$. The subfactor $L^{K} \subset L \rtimes H$ is called a Bisch-Haagerup-subfactor [5]. By [4, 7], its subfactor planar algebra is isomorphic to the fixed point space of a bipartite graph planar algebra as follows. Consider the bipartite graph $\Gamma$ where $V^{+}=\left\{v_{g H}: g H \in G / H\right\}$ is a copy of the coset space $G / H$ and $V^{-}=\left\{v_{g K}: g K \in G / K\right\}$ is a copy of the coset space $G / K$. The set of edges $C_{1}=\left\{e_{g}: g \in G\right\}$ is equal to a copy of $G$ where $e_{g}$ is an edge between $v_{g H}$ and $v_{g K}$. Let $\mu$ be the weight on $\Gamma$ that assigns $|K|^{1 / 2} /|H|^{1 / 2}$ to any edge in $C_{1}^{+}$. Let $\mathcal{P}$ be the bipartite graph planar algebra associated to $(\Gamma, \mu)$. The group $G$ acts by left multiplication on 
$(\Gamma, \mu)$ and the fixed point space $\mathcal{Q}=\mathcal{P}^{G}$ is isomorphic to the subfactor planar algebra of the subfactor $L^{K} \subset L \rtimes H$. Consider the even vertex $v_{H}$. The subgroup of $G$ that fixes $v_{H}$ is equal to $H$. By Theorem 4.5, there exists a $\mathrm{II}_{1}$ factor $A$ and a cocycle action of the Hecke pair $(G, H)$ on $A \bar{\otimes} A^{\text {op }}$ such that $H$ acts on $A$ and such that the symmetric enveloping inclusion of $\mathcal{Q}$ is isomorphic to $A^{H} \bar{\otimes}\left(A^{\mathrm{op}}\right)^{H} \subset \mathrm{vN}\left[A \bar{\otimes} A^{\mathrm{op}} ; G, H\right]$.

4.3.3. Binary trees and subfactors. Consider two non-zero natural numbers $r^{+}$and $r^{-}$. Let $\Gamma=T\left(r^{ \pm}\right)$be the infinite bipartite tree where any vertex $v \in V^{ \pm}$has degree $r^{ \pm}$. Define the weight $\mu$ such that $\mu(a)=\sqrt{r^{-} / r^{+}}$for any $a \in C_{1}^{+}$. We have that the weighted graph $(\Gamma, \mu)$ satisfies all the assumptions of Section 1 and has modulus $\delta$ equal to $\sqrt{r^{+} r^{-}}$. Let $\mathcal{P}$ be the bipartite graph planar algebra associated to $(\Gamma, \mu)$. The group $\operatorname{Aut}(\Gamma, \mu)$ is equal to the automorphism group of the bipartite graph $G=\operatorname{Aut}(\Gamma)$ which acts transitively on the even and odd vertices and on the edges. Let $o$ be an even vertex of $\Gamma$ and $G_{o}$ the subgroup of $G$ that fixes this vertex. The group $G_{o}$ is an infinite group. Let $\mathcal{Q}$ be the fixed point planar algebra under the action of $G$. It is an irreducible subfactor planar algebra. By Theorem 4.5, there exists a $\mathrm{II}_{1}$ factor $A$ and a cocycle action of the Hecke pair $\left(G, G_{o}\right)$ on $A \bar{\otimes} A^{\text {op }}$ such that $G_{o}$ acts on $A$ and such that the symmetric enveloping inclusion of $\mathcal{Q}$ is isomorphic to $A^{G_{o}} \bar{\otimes}\left(A^{\mathrm{op}}\right)^{G_{o}} \subset \mathrm{vN}\left[A \bar{\otimes} A^{\mathrm{op}} ; G, G_{o}\right]$.

Acknowledgement. Part of this work was done when the author was visiting the Institute Monsieur Matthieu in Paris during Summer 2015. He gratefully acknowledges the kind hospitality he received. The author expresses his gratitude to Cyril Houdayer and Jesse Peterson for very valuable comments and for constant support and encouragement of Dietmar Bisch and Vaughan Jones. The author was partially supported by NSF Grant DMS-1362138.

\section{REFERENCES}

[1] C. Anantharaman-Delaroche. Approximation properties for coset spaces and their operator algebras. to appear in Proc. Operator Theory, Timisoara.

[2] Y. Arano and S. Vaes. C*-tensor categories and subfactors for totally disconnected groups. In Operator algebras and Applications, The Abel Symposium 2015, pages 1-43. Springer, 2016.

[3] D. Bisch, P. Das, and S.K. Ghosh. The planar algebra of diagonal subfactors, volume 11, pages 23-47. Amer. Math. Soc., 2009.

[4] D. Bisch, P. Das, and S.K. Ghosh. The planar algebra of groupe-type subfactors. J. Funct. Anal., 257(1):20-46, 2009.

[5] D. Bisch and U. Haagerup. Composition of subfactors: new examples of infinite depth subfactors. Ann. scient. Éc. Norm. Sup., 29:329-383, 1996.

[6] A. Brothier and V.F.R. Jones. Hilbert modules over a planar algebra and the Haagerup property. $J$. Funct. Anal., 269:3634-3644, 2015.

[7] R.D. Burstein. Automorphisms of the bipartite graph planar algebra. J. Funct. Anal., 259:2384-2403, 2010.

[8] S. Curran, V.F.R. Jones, and D. Shlyakhtenko. On the symmetric enveloping algebra of planar algebra subfactors. Trans. Amer. Math. Soc, 366(1):113-133, 2014.

[9] K. De Commer and M. Yamashita. Tannaka-Krein duality for compact quantum homogeneous spaces II. classification of quantum homogeneous spaces for quantum SU(2). J. Reine Angew. Math., 708:143-171, 2015.

[10] P. Eymard. Moyennes invariantes et représentations unitaires, volume 300. Springer-Verlag, Berlin, 1972. 
[11] A. Guionnet, V.F.R. Jones, and D. Shlyakhtenko. Random matrices, free probability, planar algebras and subfactor. Quanta of maths: Non-commutative Geometry Conference in Honor of Alain Connes, in Clay Math. Proc., 11:201-240, 2010.

[12] V.F.R. Jones. Planar algebras I. Preprint. arXiv:9909.02\%.

[13] V.F.R. Jones. Index for subfactors. Invent. Math, 72:1-25, 1983.

[14] V.F.R. Jones. The planar algebra of a bipartite graph. In NJ World Sci. Publ., River Edge, editor, Knots in Hellas 98, volume 24, pages 94-117. Ser. Knots Everything, 2000.

[15] V.F.R. Jones. Planar algebra course at Vanderbilt. http://math.berkeley.edu/ vfr/, 2012.

[16] V.F.R. Jones and D. Penneys. The embedding theorem for finite depth subfactor planar algebras. Quantum Topol., 2(3):301-337, 2011.

[17] V.F.R. Jones, D. Shlyakhtenko, and K. Walker. An orthogonal approach to the subfactor of a planar algebra. Pacific J. Math., 246:187-197, 2010.

[18] V.F.R. Jones and V.S. Sunder. Introduction to subfactors. LMD, Cambridge, 1997.

[19] R. Longo and K. Rehren. Nets of subfactors. Rev. Math. Phys., 7:567-597, 1995.

[20] N. Monod and S. Popa. On co-amenability for groups and von Neumann algebras. C.R. Math. Acad. Sci. Soc. R. Can., 25(3):82-87, 2003.

[21] S. Morrison and K. Walker. Planar algebras, connections, and Turaev-Viro theory. Preprint.

[22] A. Ocneanu. Quantized groups, string algebras and Galois theory for algebras. Operator algebras and applications, London Math. Soc. Lecture Note Ser., 136:119-172, 1988.

[23] R. Palma. Crossed products by Hecke pairs I: *-completions. Preprint, arXiv:1212.5756, to appear in Mem. Amer. Math. Soc.

[24] R. Palma. Crossed products by Hecke pairs II: $C^{*}$-completions. Preprint, arXiv:1301.3320, to appear in Mem. Amer. Math. Soc.

[25] S. Popa. Correspondences. INCREST, 1986.

[26] S. Popa. Markov traces on universal Jones algebras and subfactors of finite index. Invent. Math., 111:375-405, 1993.

[27] S. Popa. Classification of amenable subfactors of type II. Acta. Math., 172:163-255, 1994.

[28] S. Popa. Symmetric enveloping algebras, amenability and AFD properties for subfactors. Math. Res. Lett., 1:409-425, 1994.

[29] S. Popa. An axiomatization of the lattice of higher relative commutants of a subfactor. Invent. Math., 120(3):427-445, 1995.

[30] S. Popa. Some properties of the symmetric enveloping algebra of a subfactor, with applications to amenability and property T. Doc. Math., 4:665-744, 1999.

[31] S. Popa. Universal construction of subfactors. J. Reine Angew. Math., 543:39-81, 2002. 